Discussion Paper No. 1005

\title{
MECHANISM DESIGN WITHOUT QUASILINEARITY
}

\author{
Tomoya Kazumura \\ Debasis Mishra \\ Shigehiro Serizawa
}

June 2017

The Institute of Social and Economic Research

Osaka University

6-1 Mihogaoka, Ibaraki, Osaka 567-0047, Japan 


\title{
MECHANISM DESIGN WITHOUT QUASILINEARITY *
}

\author{
Tomoya Kazumura, Debasis Mishra, and Shigehiro Serizawa ${ }^{\dagger}$
}

June 8, 2017

\begin{abstract}
This paper studies a model of mechanism design with transfers where agents' preferences need not be quasilinear. In such a model, (1) we characterize dominant strategy incentive compatible mechanisms using a monotonicity property; (2) we establish a revenue uniqueness result: for every dominant strategy implementable allocation rule, there is a unique payment rule that can implement it; and (3) we show that every dominant strategy incentive compatible, individually rational, and revenue-maximizing mechanism must charge zero transfer for the worst alternative (outside option). These results are applicable in a wide variety of problems (single object auction, multiple object auction, public good provision etc.) under suitable richness of type space. In particular, our results can be applied to models where preferences of agents are arbitrarily small perturbations of quasilinear preferences and illustrate the (non)-robustness of some of the classic results in mechanism design with quasilinearity. We show various applications of our results.
\end{abstract}

KEYWORDS. incentive compatibility; individual rationality; monotonicity; non-quasilinear preferences; revenue equivalence

JEL CoDE. D82, D44, D40.

${ }^{*}$ We are grateful to Sushil Bikhchandani, Juan Carlos Carbajal, Arunava Sen and participants at the Vanderbilt Mechanism Design Conference, 2016, Singapore Management University Workshop on Mechanism Design, 2016 for useful comments. We gratefully acknowledge financial support from the Joint Usage/Research Center at ISER, Osaka University and the Japan Society for the Promotion of Science (Kazumura, 14J05972; Serizawa, 15J01287, 15H03328, 15H05728). Part of this research was carried out when Debasis Mishra was visiting ISER, Osaka University. He acknowledges hospitality and support of ISER, Osaka University.

†Kazumura: Graduate School of Economics, University of Tokyo, pge003kt@gmail.com; Mishra: Indian Statistical Institute, Delhi, dmishra@isid.ac.in; Serizawa: Institute of Social and Economic Research, Osaka University, serizawa@iser.osaka-u.ac.jp 


\section{Introduction}

Standard models of mechanism design with transfers assume that agents have quasilinear preferences. This is highlighted in two recent books on this topic (Vohra, 2011; Borgers et al., 2015), which extensively describe the research frontier of mechanism design theory under the quasilinearity assumption. However, there are ample reasons to believe that agents in reallife may not have quasilinear preferences. For instance, consider the sale of a high-valued object such as spectrum bandwidth in an auction. In such a setting, bidders usually have budget constraints and often borrow from banks to pay the deficit amount. Such budget constraints make the preferences non-quasilinear. More generally, income effects in many problems lead to non-quasilinear preferences. One of the important reasons to study a model of mechanism design without quasilinearity is its appeal of being "detail-free". A model with quasilinearity assumes too much knowledge of the designer about the utility function of the agents. Hence, any result with this assumption will be vulnerable to the standard critique that the mechanism design is not detail-free.

A primary objective of this paper is to test the robustness of some of the foundational results in mechanism design to the quasilinearity assumption. We analyze a model of mechanism design with transfers where preferences of agents over transfers need not be quasilinear. In particular, agents have classical (continuous and monotonic in transfers) preferences (types) over the entire set of consumption bundles - a consumption bundle consists of an alternative and a transfer amount. Thus, we consider a model without an explicit assumption on the functional form of the utility functions of agents.

We provide two broad classes of results. The first class of results cover a variety of important problems: single object auction, multiple object auction, public good provision problem etc. If the type space has some richness, we provide a simple monotonicity condition which along with the taxation principle is necessary and sufficient for a mechanism to be (dominant strategy) incentive compatible. ${ }^{1}$ Further, we establish a revenue uniqueness result: for every implementable allocation rule, there is a unique payment rule such that the corresponding mechanism is incentive compatible. Though we do not have revenue equivalence in our model, our results can be interpreted as a counterpart of the monotonicity and revenue equivalence results for the quasilinear type spaces. ${ }^{2}$

\footnotetext{
${ }^{1}$ The taxation property simply requires that if the allocation decision at two types is the same, then the payment decision must also be the same. While this terminology is somewhat non-standard, we borrow it from Vohra (2011).

${ }^{2}$ We discuss the literature in detail later, but remind the reader that such characterization results in the quasilinear type spaces form the core for any optimization exercise (for instance, expected revenue maximization).
} 
We discuss the precise richness of type space required for our results later. One of the interesting type spaces where our results apply is the following. Take the set of all quasilinear preferences in a standard mechanism design problem (say, in a multi-object auction problem) and perturb these set of preferences to allow for small (potentially, arbitrarily small) amount of non-quasilinearity. Our monotonicity characterization and revenue uniqueness theorem apply to such type spaces. This shows how some of the benchmark results in mechanism design change even with slight perturbation of quasilinearity.

We also study the implication of individual rationality without quasilinearity. To remind, a straightforward consequence of revenue equivalence in quasilinear environment is that a $a$ revenue maximizing incentive compatible and individually rational mechanism charges zero transfer for allocating the worst alternative. We show robustness of this result in any nonquasilinear type space even though revenue equivalence may not hold.

In an application of these results, we explore how a generalization of the well known VCG mechanism may not be dominant strategy incentive compatible in type spaces with non-quasilinear preferences. We also provide a simple proof of dominant strategy incentive compatibility of a celebrated mechanism due to Demange and Gale (1985) for a particular private good provision problem.

Our second set of results work for problems where each agent has two possible alternatives to be allocated: for instance, in a single object auction problem, every agent is either allocated the object or not. Other examples include monopoly pricing, bilateral trade, deciding provision of a single public good etc. For these problems, we can relax our richness of type space to an appropriate notion of convexity. Under such convex domain of preferences, we provide an extension of the characterization in Myerson (1981) to type spaces without quasilinearity. We apply this result to some specific problems.

Our first application of this result is a problem where a seller is selling an object to a single buyer with non-quasilinear preferences. We derive the optimal (expected revenue maximizing and individually rational) incentive compatible mechanism for this problem. Even though the preference of the buyer is non-quasilinear, the optimal mechanism is similar to the quasilinear case. In the optimal mechanism, the seller posts a (monopoly) reserve price (based on the prior) and the buyer buys the object if his willingness to pay is higher than the reserve price. If the willingness to pay is less than the reserve price, then the buyer does not buy the object and no transfers are made. We emphasize that these results are obtained even though we do not have revenue equivalence in this framework.

We then analyze the problem of finding an expected revenue maximizing mechanism when there are multiple buyers. Unlike the single buyer case, this problem becomes intractable 
to analyze. In a restricted class of mechanisms, we show some qualitative properties of the optimal mechanism by using our two-alternative characterization.

Finally, we apply our results to the design of incentive compatible and anonymous mechanisms for allocating a single indivisible object among a set of agents. Anonymity requires a minimal amount of fairness. It is a compelling desiderata in fair allocation literature (Thomson, 2016). Under a mild condition on the payment of losing agents (agents who are not allocated the object), we provide a complete characterization of incentive compatible and anonymous mechanisms. These mechanisms are analogues of the Vickrey auction with agentspecific reserve prices - see a related auction and its analysis in Yamashita (2015) for the quasilinear model. Our characterization provides an axiomatic treatment of this class of mechanisms using our results.

The rest of the paper is organized as follows. We introduce our model in Section 2 and define our type space in Section 3. We present our general results on monotonicity and revenue uniqueness in Section 4. We present specific results for the two alternatives case in Section 4.2 and compare our results to results with quasilinear preferences in Section 4.3. We present the implications of incentive compatibility and individual rationality in Section 5. Section 6 presents various applications of our results. We discuss the connection of our work with the literature in Section 7. We conclude in Section 8. All our missing proofs are

in the Appendix A at the end. Appendix B is supplementary and contains discussions and preliminary results on optimal single object auction design with many agents.

\section{The model}

Let $A$ be a finite set of alternatives. The (consumption) bundles are $\mathcal{Z}=A \times \mathbb{R}$, where a typical element $z \equiv(a, t)$ corresponds to alternative $a \in A$ and transfer $t \in \mathbb{R}$. Throughout the paper, $t$ will be interpreted as the amount paid by an agent to the designer, i.e., a negative $t$ will indicate that the agent receives a transfer of $-t$.

There is a single agent and his preference over $\mathcal{Z}$ is defined by a preference ordering over $\mathcal{Z}$. The single agent assumption is for convenience and all our results extend to the multiple agents case with dominant strategy as the solution concept. For any preference ordering $R$ over $\mathcal{Z}$, we denote its strict part as $P$ and the indifference part as $I$. A preference ordering $R$ over $\mathcal{Z}$ is classical if it satisfies the following assumptions:

1. Money monotonicity. for every $t>t^{\prime}$ and for every $a \in A$, we have $\left(a, t^{\prime}\right) P(a, t)$.

2. Continuity. for every $z \in \mathcal{Z}$, the sets $\left\{z^{\prime} \in \mathcal{Z}: z^{\prime} R z\right\}$ and $\left\{z^{\prime} \in \mathcal{Z}: z R z^{\prime}\right\}$ are closed. 
3. Possibility of compensation. for every $z \in \mathcal{Z}$ and for every $b \in A$, there exists $t$ and $t^{\prime}$ such that $z R(b, t)$ and $\left(b, t^{\prime}\right) R z$.

A quasilinear preference is classical. In particular, a preference $R$ is quasilinear if there exists $v \in \mathbb{R}^{|A|}$ such that for every $a, b \in A$ and $t, t^{\prime} \in \mathbb{R},(a, t) R\left(b, t^{\prime}\right)$ if and only if $v_{a}-t \geq v_{b}-t^{\prime}$. Usually, $v$ is referred to as the valuation of the agent. The idea of valuation may be generalized as follows for classical preferences.

Definition 1 The valuation of the agent with preference $R$ for alternative $a \in A$ at bundle $z \in \mathcal{Z}$ is defined as $V^{R}(a, z)$, which uniquely solves

$$
\left(a, V^{R}(a, z)\right) I z
$$

So, $V^{R}(a, z)$ is the amount of transfer needed to make the agent (with preference $R$ ) indifferent between consuming $a$ with this transfer and $z$. An illustration of the valuation is shown in Figure 1. In the figure, the two horizontal lines correspond to two alternatives

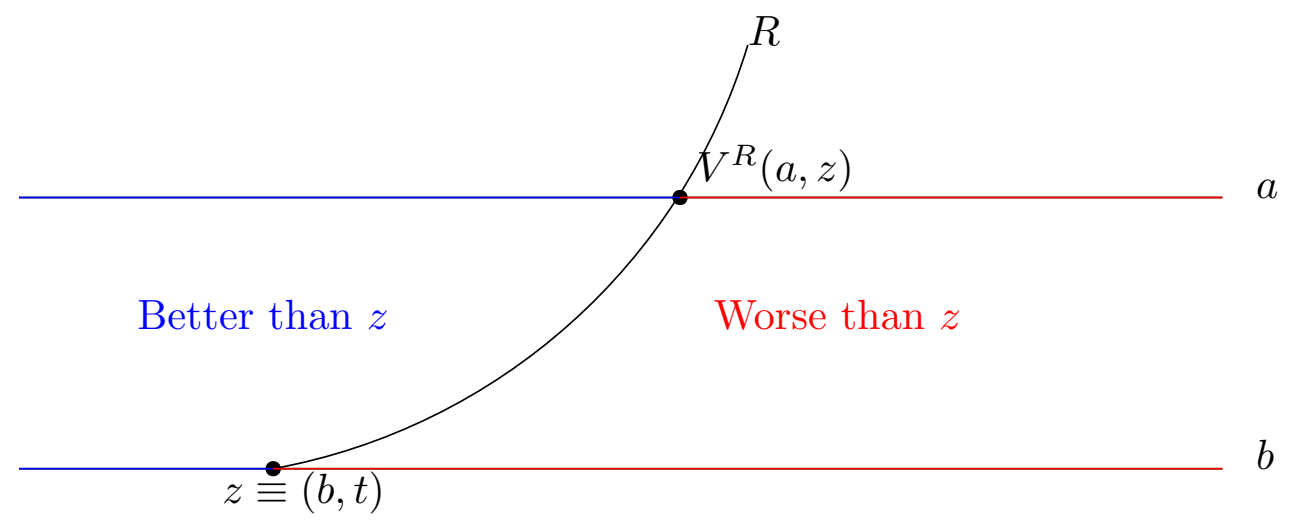

Figure 1: Valuation at a preference

$a$ and $b$. The horizontal lines indicate transfer amounts. Hence, the two lines are the entire set of consumption bundles of the agent. A preference $R$ can be described by drawing (non-intersecting) indifference vectors through these consumption bundles (lines). One such indifference vector passing through $z$ is shown in Figure 1. This indifference vector actually consists of two points $-z \equiv(b, t)$ and $\left(a, V^{R}(a, z)\right)$ as shown. Part of the curve in Figure 1 which lies outside the two consumption bundle lines is useless and has no meaning - it is only displayed for convenience. Since we will use such figures throughout the paper, it is important to clarify these issues.

The next fact shows that a valuation always exists. 
FACT 1 (Kazumura and Serizawa (2016)) For every $a \in A$, for every $z \in \mathcal{Z}$, and for every classical $R$, the valuation $V^{R}(a ; z)$ exists.

Proof: By finiteness, there exist transfers $t, t^{\prime}$ such that $(a, t) R z R\left(a, t^{\prime}\right)$. By money monotonicity and continuity, there must exist a unique $t^{\prime \prime}$ such that $\left(a, t^{\prime \prime}\right) I z$.

For any $R$ and for any $z \in \mathcal{Z}$, the valuations at bundle $z$ with preference $R$ is a vector in $\mathbb{R}^{|A|}$ and will be denoted by $V^{R}(z) \equiv\left\{V^{R}(a, z)\right\}_{a \in A}$. The collection of all such (nonintersecting) indifference vectors for all $z$ describe the preference ordering $R$.

\section{Type space}

In this section, we introduce our type space by specifying the richness required for our results. Later, in Section 4, we establish our main results on this type space. Later, we show how the richness required can be relaxed if $|A|=2$.

\subsection{Order respecting preferences}

Fix a vector $v \in \mathbb{R}^{|A|}$. For every $a, b \in A$, if $\left(a, v_{a}\right) I\left(b, v_{b}\right)$, we say $v$ belongs to an indifference class of preference $R$. The set of all vectors in $\mathbb{R}^{|A|}$ which form an indifference class of preference $R$ is denoted by $\mathcal{I}(R)$. An equivalent way to describe $R$ is to describe $\mathcal{I}(R)$. Note that the elements of $\mathcal{I}(R)$ are distinct component-wise, i.e., for all $v, v^{\prime} \in \mathcal{I}(R)$, $v_{a} \neq v_{a}^{\prime}$ for all $a \in A$. Further, the elements of $\mathcal{I}(R)$ can be ordered (since indifference vectors do not intersect), i.e., for every $v, v^{\prime} \in \mathcal{I}(R)$, we have $v_{a}>v_{a}^{\prime}$ if and only if $v_{b}>v_{b}^{\prime}$ for all $a, b \in A$.

In almost all mechanism design problems, there is an exogenous preference over alternatives. For instance, in the multi-unit auction problem, getting $k$ units at a price is preferred to getting $k^{\prime}<k$ units at the same price (assuming free disposal). One way to model this is to impose an exogenous ordering on the set of alternatives such that the alternative of getting $k$ units is strictly preferred to $k^{\prime}$ units. To cover such large class of problems in our type space, we assume that there is a partial order $\succ$ on the set of alternatives $A$. A vector $v \in \mathbb{R}^{|A|}$ respects $\succ$ if for every $a, b \in A$, we have

$$
a \succ b \Rightarrow v_{a}>v_{b} .
$$

Definition 2 A preference relation $R$ respects a strict partial order $\succ$ if every $v \in \mathcal{I}(R)$ respects $\succ$. A domain $\mathcal{R}$ of preference relations is order respecting if there exists $\succ$ such that every $R \in \mathcal{R}$ respects $\succ$. 
We will usually denote an order respecting domain as $\mathcal{R}^{\succ}$ to indicate that every $R \in \mathcal{R}^{\succ}$ respects the partial ordering $\succ$.

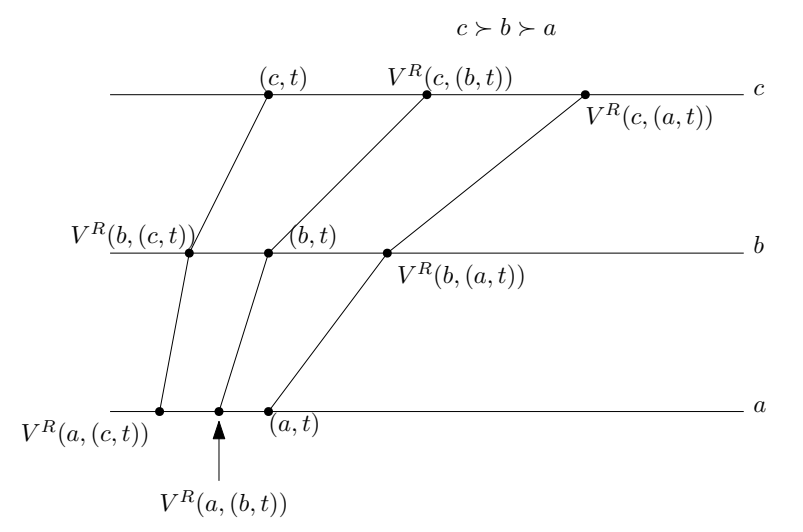

Figure 2: Indifference vectors in order respecting domain

An equivalent way to state this domain restriction is the following. Consider $R$ which respects $\succ$ and pick $a \in A$ and $t \in \mathbb{R}$. Then, for every $b \in A$, we have $V^{R}(b,(a, t))<t$, if $a \succ b$. Hence, a domain $\mathcal{R}$ is order respecting if there exists $\succ$ such that for every $R \in \mathcal{R}$, for every $t \in \mathbb{R}$ and every $a, b \in A$, we have

$$
a \succ b \text { implies } V^{R}(b,(a, t))<t .
$$

In particular, if we arrange the alternatives according to the specified ordering $\succ$ from bottom to top, the points along the indifference vector shift to the right as we move along $\succ$. This is illustrated in Figure 2 for three alternatives and the relation $\succ$ defined as $c \succ b \succ a$ (though $\succ$ is shown to be a complete relation here, this need not be the case). The preference relation $R$, whose indifference vectors are shown in Figure 2, respects $\succ$.

Order respecting domains capture many interesting problems. They were introduced in Bikhchandani et al. (2006) for quasilinear domains, and ours is an extension to nonquasilinear domains. ${ }^{3}$ We list their applicability below:

- $\succ$ is empty. Then, there are no restrictions in preferences over alternatives. This captures public decision problems, where the set of alternatives is a set of public projects $-\succ$ being empty represents the fact that there is no restriction on the preferences over public projects at a given transfer level.

\footnotetext{
${ }^{3}$ There is a minor difference between our definition and the definition in Bikhchandani et al. (2006) they allow for indifferences in $\succ$ but we do not allow indifferences. We make this assumption for technical reasons.
} 
- $\succ$ is a complete ordering. This can capture a situation where there is a set of homogeneous objects (set of alternatives in this example) and there is no restriction on the number of units that an agent can consume. More units are better for the agents. So, alternatives can be completely ranked by the number of units. The complete ordering can also capture common-object-ranking preferences in multi-object allocation problem with unit demand (Zhou and Serizawa, 2016).

- $\succ$ is a partial ordering. This can capture multiple heterogeneous objects sale (set of alternatives in this example), where agents can have unit demand (Morimoto and Serizawa, 2015) or multiple demand as in combinatorial auctions with package bidding (Ausubel and Milgrom, 2002). If they have unit demand, then the only restriction is that every object is strictly preferred to no object. If agents demand more than one object, then the only restriction is that a subset of a set of objects is less preferred than the original set of objects. The partial ordering can also capture "common-tieredobject-ranking" preferences in the unit demand model of Zhou and Serizawa (2016).

\subsection{Richness}

Our results will work in order respecting domains satisfying some amount of richness. To understand our richness, first consider the set of quasilinear preferences respecting a partial order $\succ$ - denote this as $\mathcal{Q}^{\succ}$. A typical preference $R$ in $\mathcal{Q}^{\succ}$ is shown in Figure 3, where we see that the indifference vectors of $R$ are "parallel" to each other. In particular, if we consider $v, \hat{v} \in \mathcal{I}(R)$, then $\left(v_{x}-v_{y}\right)-\left(\hat{v}_{x}-\hat{v}_{y}\right)=0$ for all $x, y \in A$. Hence, the domain $\mathcal{Q}^{\succ}$ satisfies the richness that for any two vectors $v, \hat{v}$ which are "parallel" to each other and respect $\succ$, there is a preference relation $R \in \mathcal{Q}^{\succ}$ such that $v, \hat{v} \in \mathcal{I}(R)$.

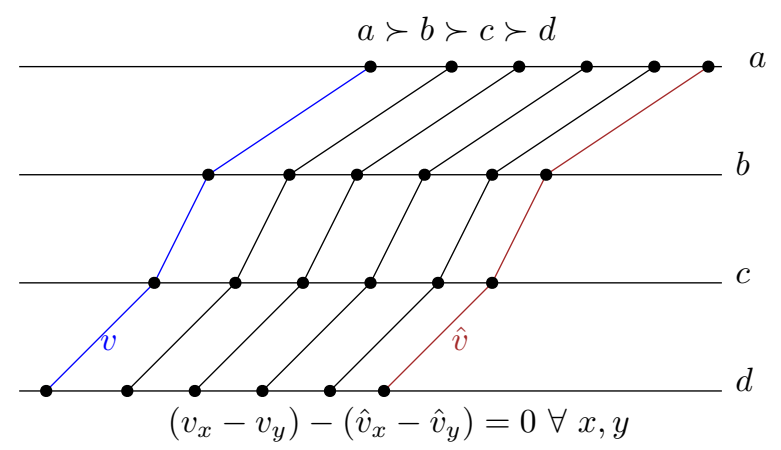

Figure 3: A quasilinear preference

Our richness requires a slight strengthening of this idea. Call two vector $v, \hat{v} \in \mathbb{R}^{|A|}$ as 
$\epsilon$-parallel for some $\epsilon>0$ if for every $x, y \in A$, we have

$$
\left|\left(v_{x}-v_{y}\right)-\left(\hat{v}_{x}-\hat{v}_{y}\right)\right|<\epsilon .
$$

We say $v, \hat{v} \in \mathbb{R}^{|A|}$ are pairwise consistent for $\succ$ if (a) $v, v^{\prime}$ respect $\succ$ and (b) either $v>\hat{v}$ or $v<\hat{v}$.

Suppose $v$ and $\hat{v}$ are pairwise consistent and $\epsilon>0$ is very close to zero, then $v$ and $\hat{v}$ are $\epsilon$-parallel means that they are "close to" parallel. In general, we will not require $\epsilon$ to be close to zero.

Definition 3 An order respecting domain $\mathcal{R}^{\succ}$ satisfies $\epsilon$-two-point $\left(\mathbf{T P}^{\epsilon}\right)$ richness for some $\epsilon>0$ if for every pair of $\epsilon$-parallel $v, v^{\prime} \in \mathbb{R}^{|A|}$ which are pairwise consistent for $\succ$, there is a preference $R \in \mathcal{R}^{\succ}$ such that $v, v^{\prime} \in \mathcal{I}(R)$.

Our main results will use this richness in order respecting domains. The quasilinear domain of preferences $\mathcal{Q}^{\succ}$ does not satisfy this richness since it does not have enough variety of preferences required by $\mathrm{TP}^{\epsilon}$ richness. Later, we give specific examples of order respecting domains that satisfy $\mathrm{TP}^{\epsilon}$ richness - this includes domains which are arbitrarily small perturbations of $\mathcal{Q}^{\succ}$. Below, we illustrate $\mathrm{TP}^{\epsilon}$ richness further though an example.

Pick any $v$ and $\hat{v}$ which are pairwise consistent and $\epsilon$-parallel for some $\epsilon>0$. First, it is easy to see that there are infinitely many preferences $R$ respecting $\succ$ such that $v, \hat{v} \in \mathcal{I}(R)$ - this is because we have infinitely many ways to choose other indifference vectors. Our condition $\mathrm{TP}^{\epsilon}$ richness requires just one preference $R$ respecting $\succ$ to be part of the domain such that $v, \hat{v} \in \mathcal{I}(R)$. Next, we construct one specific preference $\bar{R}$ respecting $\succ$ and satisfying $v, \hat{v} \in \mathcal{I}(\bar{R})$ - this is obviously not the only way to satisfy $\mathrm{TP}^{\epsilon}$ richness.

The preference $\bar{R}$ is constructed by specifying the indifference vectors of $\bar{R}$. For all $v^{\prime \prime}<v$, we have $v^{\prime \prime} \in \mathcal{I}(\bar{R})$ if and only if $v_{x}^{\prime \prime}-v_{y}^{\prime \prime}=v_{x}-v_{y}$ for all $x, y \in A$. For all $v^{\prime \prime}>\hat{v}$, we have $v^{\prime \prime} \in \mathcal{I}(\bar{R})$ if and only if $v_{x}^{\prime \prime}-v_{y}^{\prime \prime}=\hat{v}_{x}-\hat{v}_{y}$ for all $x, y \in A$. For all $v \leq v^{\prime \prime} \leq \hat{v}$, we have $v^{\prime \prime} \in \mathcal{I}(\bar{R})$ if and only if there exists $\alpha \in[0,1]$ such that $v^{\prime \prime}=\alpha v+(1-\alpha) \hat{v}$. Informally, indifference vectors to the left of $v$ are parallel to $v$, to the right of $\hat{v}$ are parallel to $\hat{v}$, and it is a "convex combination" of indifference vectors of $v$ and $\hat{v}$ between $v$ and $\hat{v}$. It is clear that $\bar{R}$ exists and respects $\succ$. A pictorial description of $\bar{R}$ is given in Figure 4 .

\section{Monotonicity and incentive compatibility}

We now define the notion of incentive compatibility we use. For the one agent model, we refer to it as simply incentive compatibility, but when this is extended to the multi-agent model, we will be using dominant strategy incentive compatibility. 


$$
d \succ b \succ c \succ a
$$

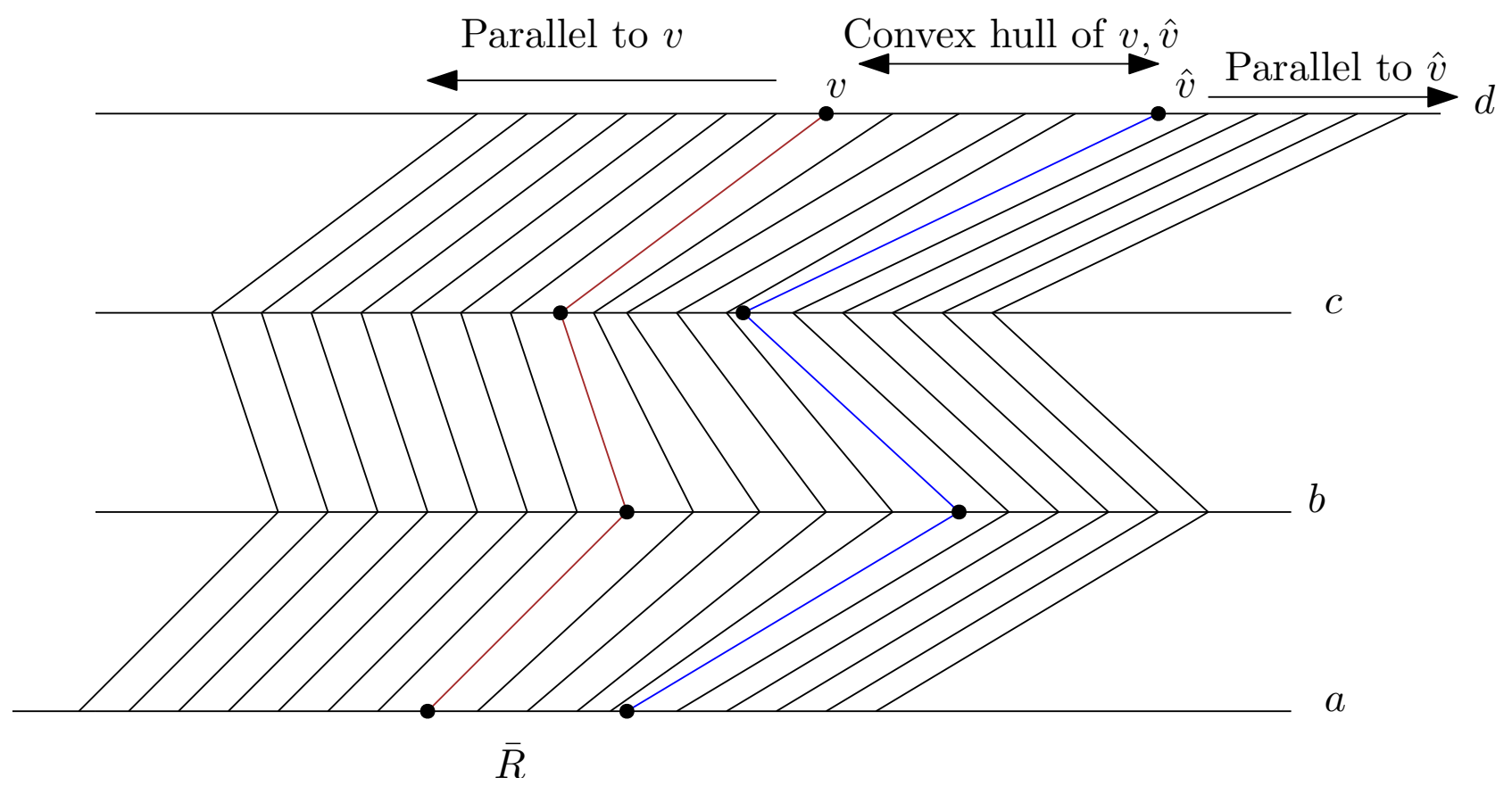

Figure 4: $\mathrm{TP}^{\epsilon}$ richness - construction of $\bar{R}$

\subsection{Incentive compatibility and revenue uniqueness}

Let $\mathcal{R}$ be any subset of classical preferences - we will refer to $\mathcal{R}$ as the domain. A mechanism $(f, p)$ is a pair, where $f: \mathcal{R} \rightarrow A$ and $p: \mathcal{R} \rightarrow \mathbb{R}$. Throughout, we will assume that $f$ is onto - most of the results and their proofs can be modified in a straightforward way if $f$ is not onto.

The mechanisms we consider here are direct mechanisms and restriction to such mechanisms is due to the revelation principle.

Definition 4 A mechanism $(f, p)$ is incentive compatible if for every $R, \hat{R} \in \mathcal{R}$, we have

$$
(f(R), p(R)) R(f(\hat{R}), p(\hat{R})) .
$$

The following monotonicity property will be shown to be necessary for incentive compatibility.

Definition 5 A mechanism $(f, p)$ is monotone if for every $R, \hat{R} \in \mathcal{R}$ with $f(R) \equiv a$ and $(f(\hat{R}), p(\hat{R})) \equiv z$, we have

$$
V^{R}(a, z) \geq V^{\hat{R}}(a, z) .
$$


An illustration is shown in Figure 5. Take any pair of preferences $R$ and $\hat{R}$ and suppose the

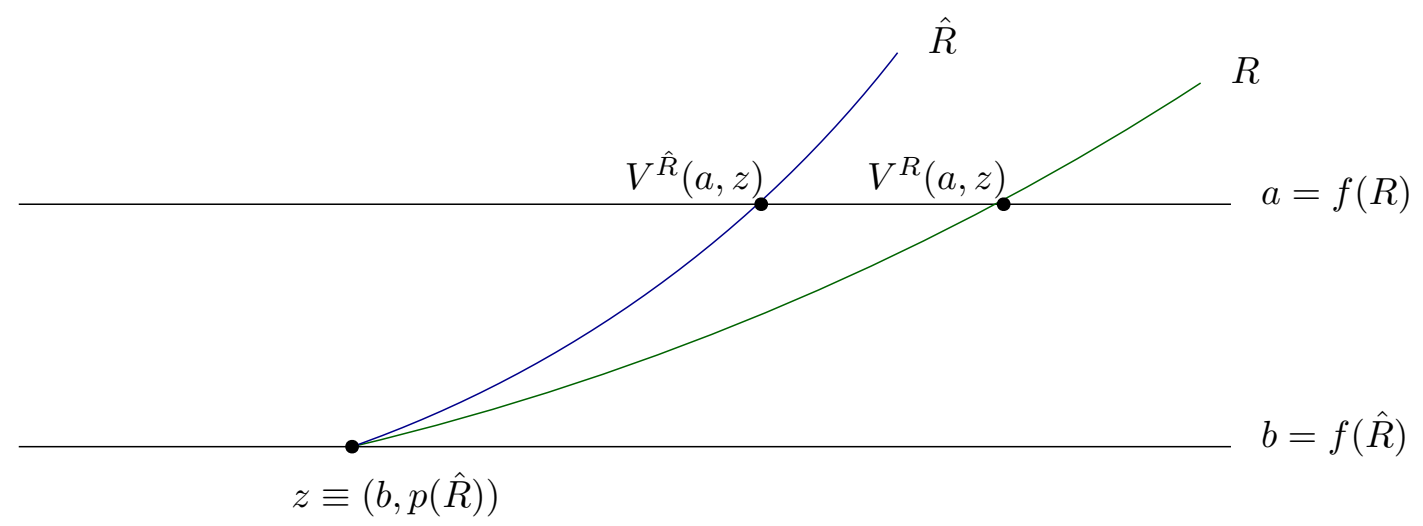

Figure 5: Illustration of monotonicity

mechanism chooses consumption bundle $z$ at $\hat{R}$. Then, monotonicity requires that valuation for $f(R)$ at $z$ is larger for $R$ than for $\hat{R}$. There is another natural way to interpret the monotonicity condition. Suppose the mechanism chooses the alternative $a$ at $R$. Now, consider another preference $\hat{R}$ such that $V^{\hat{R}}(a, z)>V^{R}(a, z)$, where $z$ does not involve alternative $a$. In other words, agent's liking for the outcome at $R$ compared to $z$ increases from $R$ to $\hat{R}$. Then, monotonicity requires $(f(\hat{R}), p(\hat{R})) \neq z$.

As we show later, this monotonicity condition collapses to the standard weak monotonicity or 2-cycle monotonicity condition if the domain is the quasilinear type space - see Section 4.3 for detailed discussions. We show next that monotonicity along with a simple condition on payments is equivalent to incentive compatibility.

We present this simple necessary condition for incentive compatibility next. It is usually referred to as the "taxation principle", and states that whenever the allocation decision of a mechanism is the same at two preferences, the payment decision has to be the same.

Definition 6 A mechanism $(f, p)$ satisfies the taxation principle if for every $R, R^{\prime}$ with $f(R)=f\left(R^{\prime}\right)$, we have $p(R)=p\left(R^{\prime}\right)$.

An alternate way to state the taxation principle is the following.

FACT 2 A mechanism $(f, p)$ satisfies the taxation principle if and only if there exists a map $\kappa: A \rightarrow \mathbb{R}$ such that for every $R \in \mathcal{R}^{\succ}$, we have $p(R)=\kappa(f(R))$.

It is clear that incentive compatibility implies the taxation principle. We show below monotonicity and taxation principle are sufficient for incentive compatibility. 
TheOREM 1 Suppose $\mathcal{R}^{\succ}$ is an order respecting domain satisfying TP ${ }^{\epsilon}$ richness for some $\epsilon>0$, where $\succ$ is some strict partial order over $A$. Let $(f, p)$ be a mechanism defined over $\mathcal{R}^{\succ}$. Then, the following statements are equivalent.

1. $(f, p)$ is incentive compatible.

2. $(f, p)$ is monotone and satisfies taxation principle.

Theorem 1 is the counterpart of monotonicity based characterizations found in the quasilinear domains - we discuss the precise connection in Section 4.3. Roughly, the counterpart of Theorem 1 will require monotonicity along with a revenue equivalence (envelope) formula, which is much stronger than taxation principle. In other words, in Theorem 1, we succeeded to show that monotonicity and a weaker requirement of taxation principle are sufficient to obtain incentive compatibility in domains satisfying $\mathrm{TP}^{\epsilon}$ richness.

Since quasilinearity plays a crucial role to derive revenue equivalence formula, one might expect that revenue equivalence does not hold without quasilinearity. In fact, we show that revenue uniqueness holds.

Theorem 2 Suppose $\mathcal{R}^{\succ}$ is an order respecting domain satisfying TP ${ }^{\epsilon}$ richness for some $\epsilon>0$, where $\succ$ is some strict partial order over $A$. If $(f, p)$ and $\left(f, p^{\prime}\right)$ are two incentive compatible mechanisms defined on $\mathcal{R}^{\succ}$, then $p=p^{\prime}$.

The quasilinear type space does not satisfy $\mathrm{TP}^{\epsilon}$ richness, and hence, Theorems 1 and 2 do not apply to quasilinear type space. However, these theorems help us understand the structure of incentive compatible mechanisms if the designer does not know the precise nature preferences of the agent over consumption bundles.

In Section 6, we give some applications of Theorems 1 and 2 to specific problems.

\subsection{Incentive compatibility with two alternatives}

In this section, we focus on the case where $|A|=2$. Besides covering a wider variety of applications, the two alternatives setting is important because it corresponds to a canonical class of models in mechanism design studied under quasilinearity assumption. Under quasilinearity, if there are two alternatives, valuation of one of the alternatives can always be scaled to zero and the only private information of an agent is his valuation for the other alternative. As a result, the type of an agent becomes one-dimensional. One-dimensional type spaces are ubiquitous in mechanism design literature, and their study parallels the single object auction 
analysis in Myerson (1981). Hence, analysis of the two alternatives case can be interpreted as an extension of the one-dimensional mechanism design to non-quasilinear preferences.

For simplicity, we represent the set of alternatives as $A=\{0,1\}$. Given a classical preference relation $R$, we define the willingness to pay of an agent at transfer $t$ as

$$
W P(R, t):=V^{R}(1 ;(0, t))-t
$$

Notice that the reference consumption bundle is $(0, t)$ - hence, willingness to pay $W P(R, t)$ is the extra transfer needed to make the agent indifferent between $(0, t)$ and $(1, t+W P(R, t))$. Since $V^{R}$ exists, $W P(R, \cdot)$ exists.

Definition $7 A$ domain of preferences $\mathcal{R}$ satisfies $\mathbf{W P}$-convexity if for every $t \in \mathbb{R}$, the set $\{W P(R, t): R \in \mathcal{R}\}$ is an interval.

We allow for the fact that the interval $\{W P(R, t): R \in \mathcal{R}\}$ is open - for instance, $\mathbb{R}$ or $\mathbb{R}_{++}$. WP-convexity property is a much weaker requirement than the $\mathrm{TP}^{\epsilon}$ richness condition used in Theorem 1. For instance, the set of all quasilinear preferences satisfy WP-convexity, but it will not satisfy $\mathrm{TP}^{\epsilon}$ richness. Further, note that we do not need order respecting domains here. We now provide a characterization which is closer to the standard Myersonian results in quasilinear domains.

Theorem 3 Suppose $A=\{0,1\}$ and $\mathcal{R}$ satisfies WP-convexity. If $(f, p)$ is a mechanism defined on $\mathcal{R}$, then the following statements are equivalent.

1. $(f, p)$ is incentive compatible.

2. There exists a real number $\tau^{0}$ such that

(a) WP-monotonicity. for every $R, R^{\prime} \in \mathcal{R}$ with $f(R)=1$, we have

$$
\left[W P\left(R^{\prime}, \tau^{0}\right)>W P\left(R, \tau^{0}\right)\right] \Rightarrow f\left(R^{\prime}\right)=1
$$

(b) Payment formula. for every $R \in \mathcal{R}$,

$$
p(R)=\tau^{0}+f(R) \cdot \tau^{1}
$$

where $\tau^{1}:=\inf \left\{W P\left(R^{\prime}, \tau^{0}\right): R^{\prime} \in \mathcal{R}, f\left(R^{\prime}\right)=1\right\}$.

We make some remarks about Theorem 3. 
1. Though Condition 2(b) in Theorem 3 is stronger than Condition 2(b) in Theorem 1, WP-monotonicity is weaker than monotonicity. We discuss this issue in detail in Section 4.3, where we also emphasize the fact that WP-monotonicity and monotonicity are equivalent in quasilinear type space.

2. Theorem 3 hints at dramatic reduction in the informational requirement of mechanisms in the two-alternatives model. Every mechanism must announce a pair of numbers - $\tau^{0}$ and $\tau^{1}$. Then, it only elicits $W P\left(R, \tau^{0}\right)$ information from the agent. If $W P\left(R, \tau^{0}\right)>\tau^{1}$, then the object is allocated at price $\tau^{0}+\tau^{1}$. If $W P\left(R, \tau^{0}\right)<\tau^{1}$, then the object is not allocated and the agent pays $\tau^{0}$.

3. Condition 2(b) should not be confused as a revenue equivalence result. This is because the monotonicity condition depends on the choice of $\tau^{0}$. To be more precise, suppose we have an incentive compatible mechanism $(f, p)$ defined by $f$ and $\tau^{0}$, then $\tau^{1}$ and $p$ are determined by these two parameters. If we fix $f$ and consider $\bar{\tau}^{0}:=\tau^{0}+\Delta$ for some $\Delta \in \mathbb{R}$, does that define a new incentive compatible mechanism? The answer is not clear. This is because though $f$ is satisfies monotonicity at $\tau^{0}$, it may fail to be monotone at $\bar{\tau}^{0}$. This is not an issue in the quasilinear type space because WP values do not depend on transfer amount, and hence, if $f$ satisfies monotonicity at $\tau^{0}$, it also satisfies at $\bar{\tau}^{0}$.

To highlight the last remark further, we show below that in the two-alternative model, the revenue uniqueness result can be restored under different richness requirements than Theorem 2. To do so, we introduce the notion of positive income effect. We define it using the notion of willingness to pay. As transfer increases, positive income effect requires that willingness to pay must decrease.

Definition 8 A classical preference $R$ exhibits positive income effect if for all $t, t^{\prime} \in \mathbb{R}$ with $t>t^{\prime}$, we have $W P\left(R, t^{\prime}\right)>W P(R, t)$.

The set of all classical types with positive income effect is denoted as $\mathcal{R}^{++}$.

We now state the revenue uniqueness result under positive income effect.

Theorem 4 Suppose $A=\{0,1\}$ and $\mathcal{R} \supseteq \mathcal{R}^{++}$. If $(f, p)$ and $\left(f, p^{\prime}\right)$ are incentive compatible mechanisms then $p=p^{\prime}$. 


\subsection{Comparison to quasilinear type space}

We now formally compare our results to analogous results in the quasilinear type space. The comparison below highlights how our results extend and generalize existing results with quasilinearity. As defined earlier, a quasilinear preference can be represented by a valuation vector in $\mathbb{R}^{|A|}$. Denote the valuation attached to quasilinear preferences $R, R^{\prime}, R^{\prime \prime}, \ldots$ as $v, v^{\prime}, v^{\prime \prime}, \ldots$ respectively. Let $\mathcal{V} \subseteq \mathbb{R}^{|A|}$ be the domain of valuations in the quasilinear preference domain. Notice that two valuation vectors which differ by the same constant in each component represent the same preference over consumption bundles.

In describing the results from the quasilinear domain literature, we assume that $A$ is finite and the mechanisms are deterministic. But some of the results stated below also hold for non-finite $A$ and randomized mechanisms.

Monotonicity on quasilinear domain. Our monotonicity condition reduces to the following condition in quasilinear domains. Take two valuation vectors $v, v^{\prime} \in \mathbb{R}^{|A|}$ and denote the underlying quasilinear preferences corresponding to them as $R, R^{\prime}$ respectively. Consider a mechanism $(f, p)$ and denote $f(R)=a$ and $f\left(R^{\prime}\right)=b$. Note that

$$
V^{R}\left(a,\left(b, p\left(R^{\prime}\right)\right)\right)=v_{a}-v_{b}+p\left(R^{\prime}\right) \text { and } V^{R^{\prime}}\left(a,\left(b, p\left(R^{\prime}\right)\right)\right)=v_{a}^{\prime}-v_{b}^{\prime}+p\left(R^{\prime}\right) .
$$

Hence, our monotonicity condition is: $V^{R}\left(a,\left(b, p\left(R^{\prime}\right)\right)\right) \geq V^{R^{\prime}}\left(a,\left(b, p\left(R^{\prime}\right)\right)\right)$, which is equivalent to requiring $v_{a}-v_{b} \geq v_{a}^{\prime}-v_{b}^{\prime}$. This is the familiar (weak) monotonicity or 2-cycle monotonicity condition from the quasilinear domain literature (Bikhchandani et al., 2006; Saks and Yu, 2005; Ashlagi et al., 2010). Hence, in quasilinear domain, monotonicity of the mechanism reduces to the monotonicity of the allocation rule $f$.

Mechanism characterization. The monotonicity condition becomes too weak in the quasilinear domain, and hence, the taxation principle needs to be significantly strengthened to be sufficient to imply incentive compatibility. We report two results from quasilinear domains to compare them with our results. The first result is due to Jehiel et al. (1999) and Myerson (1981) (for the single object auction environment) - we report a version of this result given in Ashlagi et al. (2010), which can be shown using subgradient techniques of convex functions (Rockafellar, 1970). To state this fact, we slightly abuse notation to write a mechanism $(f, p)$ on a quasilinear type space with domain of valuations $\mathcal{V} \subseteq \mathbb{R}^{|A|}$ as maps: $f: \mathcal{V} \rightarrow A$ and $p: \mathcal{V} \rightarrow \mathbb{R}$. 
FACT 3 (Jehiel et al. (1999); Ashlagi et al. (2010)) Suppose $(f, p)$ is a mechanism defined on a convex domain of valuations $\mathcal{V} \subseteq \mathbb{R}^{|A|}$. Then, the following statements are equivalent.

1. $(f, p)$ is incentive compatible.

2. (a) $f$ is monotone.

(b) for every $v, v^{\prime} \in \mathcal{V}$, we have

$$
p(v)=p\left(v^{\prime}\right)+\left[v_{f(v)}-v_{f\left(v^{\prime}\right)}^{\prime}\right]-\int_{0}^{1} \psi^{v^{\prime}, v}(z) d z,
$$

where $\psi^{v^{\prime}, v}(z)=\left(v-v^{\prime}\right) \cdot \mathbb{I}_{f\left(v^{\prime}+z\left(v-v^{\prime}\right)\right)}$ for all $z \in[0,1]$ and for every $v^{\prime \prime} \in \mathcal{V}, \mathbb{I}_{f\left(v^{\prime \prime}\right)}$ is the indicator vector in $\mathbb{R}^{|A|}$, where the component corresponding to $f\left(v^{\prime \prime}\right)$ is set to 1 and all other components are zero.

Fact 3 generalizes analogous results for two alternatives case in Myerson (1981) - Myerson develops this result in the single object auction framework. Statement 2(b) implies that if we have two incentive compatible mechanisms with the same allocation rule, $(f, p)$ and $\left(f, p^{\prime}\right)$, then $p(v)=p^{\prime}(v)$ for some $v$ implies that $p=p^{\prime}$. This is usually referred to as the revenue equivalence formula or the envelope formula (Milgrom and Segal, 2002; Krishna and Maenner, 2001). Contrast this to our Theorem 2, which states that in order respecting non-quasilinear type spaces satisfying $\mathrm{TP}^{\epsilon}$-richness for some $\epsilon>0$, we have revenue uniqueness, i.e., if we have two incentive compatible mechanisms $(f, p)$ and $\left(f, p^{\prime}\right)$ using the same allocation rule, then $p=p^{\prime}$. Thus, the conclusion of Theorem 2 is significantly stronger than revenue equivalence.

We can contrast our Theorem 1 with Fact 3 . Theorem 1 characterizes incentive compatibility by monotonicity of mechanism and the taxation principle. On the other hand, Fact 3 characterizes incentive compatibility using monotonicity of allocation rule and the revenue equivalence formula, which is significantly stronger than the taxation principle. The richness of our non-quasilinear type space allows us to use the monotonicity of the mechanism along with a simple condition on payments in Theorem 1 to characterize incentive compatibility. Later, we show that arbitrarily small perturbation of quasilinear type space satisfies our richness condition. Theorems 1, 2, and Fact 3 show how the results change with such arbitrarily small perturbations.

Allocation rule characterization. In the quasilinear domain, due to the separability (and linearity) of the payment from the allocation rule, one can focus attention on the implementability question. 
Definition 9 An allocation rule $f$ is implementable if there exists a payment rule $p$ such that $(f, p)$ is incentive compatible.

In quasilinear domain, if we know that $f$ is implementable, then due to Fact 3 , there is an explicit way to compute payments using $f$ (up to an additive constant). The following fact answer the question: when is an allocation rule implementable?

FACT 4 (Saks and Yu (2005); Ashlagi et al. (2010)) Suppose closure of $\mathcal{V}$ is convex and $f$ is an onto allocation rule defined on $\mathcal{V}$. Then, $f$ is implementable if and only if it is monotone.

Fact 4 was first shown for order respecting quasilinear domains in Bikhchandani et al. (2006). Though Theorem 1 seems like an extension of their result to non-quasilinear domains, it should not be confused as an analogue of Fact 4. There are two important differences: (1) Theorem 1 is a characterization of incentive compatible mechanisms using monotonicity of the mechanism and Fact 4 is a characterization of implementable allocation rules in quasilinear domains using monotonicity of allocation rule; (2) Theorem 1 uses richness of non-quasilinear domain and it does not apply to quasilinear domains.

Monotonicity versus WP-monotonicity. The monotonicity condition is somewhat symmetric in quasilinear domain. Consider the same example as above. For $R$ and $R^{\prime}$ with $f(R)=a, f\left(R^{\prime}\right)=b$, we have a pair of monotonicity conditions:

$$
\begin{aligned}
& V^{R}\left(a,\left(b, p\left(R^{\prime}\right)\right)\right) \geq V^{R^{\prime}}\left(a,\left(b, p\left(R^{\prime}\right)\right)\right) \\
& V^{R^{\prime}}(b,(a, p(R))) \geq V^{R}(b,(a, p(R))) .
\end{aligned}
$$

In quasilinear domain, both these conditions are equivalent to:

$$
v_{a}-v_{b} \geq v_{a}^{\prime}-v_{b}^{\prime} .
$$

This equivalence of the pair of monotonicity conditions need not hold in non-quasilinear environment since $R$ and $R^{\prime}$ can be arbitrary. Note that WP-monotonicity in Theorem 3 only uses one side of monotonicity. Hence, WP-monotonicity in Theorem 3 is weaker than the monotonicity condition in Theorem 1. In quasilinear domain, monotonicity is equivalent to WP-monotonicity, which is the condition identified in Myerson (1981).

\section{Individual rationality}

In this section, we investigate the implication of individual rationality along with incentive compatibility in domain of classical preferences. We focus attention to order respecting 
domains, though the result in this section holds beyond the order respecting domains. For every $\succ$, we assume the existence of a worst alternative in $A$ according to $\succ$, and denote it by $a_{0}$. For instance, in the auctioning of one or more objects, $a_{0}$ will refer to the alternative where the agent is not assigned any object. For the public good provision problem, $a_{0}$ may refer to the alternative where no public good is provided.

We further assume that the outside option of the agent is $\left(a_{0}, 0\right)$, i.e., get the alternative $a_{0}$ at zero transfer.

Definition 10 A mechanism $(f, p)$ is individually rational if for every $R$ in the domain

$$
\left((f(R), p(R)) R\left(a_{0}, 0\right)\right.
$$

The following lemma gives necessary and sufficient conditions for individual rationality similar conditions are well known with quasilinear preferences.

Lemma 1 Suppose $(f, p)$ is an incentive compatible mechanism. It is individually rational if and only if for every $R$ with $f(R)=a_{0}$, we have $p(R) \leq 0$.

Proof: For every $R$ with $f(R)=a_{0}$, individual rationality implies that $\left(a_{0}, p(R)\right) R\left(a_{0}, 0\right)$. This implies that $p(R) \leq 0$. For the converse, consider any $R$. By ontoness of the mechanism, there is some $R^{\prime}$ such that $f\left(R^{\prime}\right)=a_{0}$. Now, note that

$$
(f(R), p(R)) R\left(a_{0}, p\left(R^{\prime}\right)\right) R\left(a_{0}, 0\right)
$$

where the first relation follows from incentive compatibility and the second relation follows from the fact that $p\left(R^{\prime}\right) \leq 0$. Hence, $(f, p)$ is individually rational.

In the quasilinear domain, a straightforward consequence of Lemma 1 is the following. Take an incentive compatible and individually rational mechanism $(f, p)$ and construct another mechanism $(f, \tilde{p})$ as follows: $\tilde{p}(R)=p(R)-\kappa\left(a_{0}\right)$ for all $R$, where $\kappa\left(a_{0}\right)$ (computed using the taxation principle) is the payment of the worst alternative $a_{0}$ in the mechanism $(f, p)$. By Lemma $1, \kappa\left(a_{0}\right)$ is non-positive, and hence, $\tilde{p}(R) \geq p(R)$ for all $R$. Further, in the quasilinear domain, $(f, \tilde{p})$ remains incentive compatible. An immediate benefit of this argument is that if we are interested in maximizing revenue among all incentive compatible and individually rational mechanisms, we can look at those mechanisms which price the worst alternative at zero.

Unfortunately, the above argument fails in non-quasilinear type space since $(f, \tilde{p})$ may not be incentive compatible without quasilinearity - for instance, in order respecting domains satisfying $\mathrm{TP}^{\epsilon}$ richness, $(f, \tilde{p})$ cannot be incentive compatible due to our revenue uniqueness 
theorem (Theorem 2). Remarkably, the following result still holds in non-quasilinear type spaces.

THEOREM 5 Let $\mathcal{R}^{\succ}$ be an order respecting domain with $a_{0}$ being the worst element according to $\succ$. For every incentive compatible and individually rational mechanism $(f, p)$ on $\mathcal{R}^{\succ}$, there is an incentive compatible and individually rational mechanism $(\tilde{f}, \tilde{p})$ on $\mathcal{R}^{\succ}$ such that

1. for every $R$ with $\tilde{f}(R)=a_{0}$, we have $\tilde{p}(R)=0$,

2. for every $R$, we have $\tilde{p}(R) \geq p(R)$.

Two important remarks are in order: (a) $f$ and $\tilde{f}$ in Theorem 5 may be quite different allocation rules - this is in contrast to quasilinear domains, where we could construct $(\tilde{f}, \tilde{p})$ such that $\tilde{f}=f$; (b) Theorem 5 does not require any assumption on richness of type space.

Theorem 5 thus assures us that even in non-quasilinear type space, if we are interested in maximizing revenue among all incentive compatible and individually rational mechanisms, we can look at those mechanisms which price the worst alternative at zero.

We formalize this implication to revenue maximizing mechanism in a general model with a domain satisfying $\mathrm{TP}^{\epsilon}$ richness now. The following lemma holds true in such domains.

Lemma 2 Suppose $\mathcal{R}^{\succ}$ satisfies TP richness. If $(f, p)$ is a mechanism defined on $\mathcal{R}^{\succ}$, then it is incentive compatible if and only if for every $R, R^{\prime} \in \mathcal{R}^{\succ}$

$$
p(R)=\inf _{\hat{R}: f(\hat{R})=f(R)} V^{\hat{R}}\left(f(R),\left(f\left(R^{\prime}\right), p\left(R^{\prime}\right)\right)\right)=\sup _{\hat{R}: f(\hat{R})=f\left(R^{\prime}\right)} V^{\hat{R}}\left(f(R),\left(f\left(R^{\prime}\right), p\left(R^{\prime}\right)\right)\right) .
$$

Now, fix the worst alternative $a_{0}$ according to $\succ$. Suppose $(f, p)$ is an incentive compatible mechanism in $\mathcal{R}^{\succ}$ (which satisfies $\mathrm{TP}^{\epsilon}$ richness). Then, by taxation principle, there is a map $\kappa: A \rightarrow \mathbb{R}$ such that $t(R)=\kappa(f(R))$ for all $R \in \mathcal{R}^{\succ}$. Lemma 2 then implies that for any $a \neq a_{0}$, we must have

$$
\kappa(a)=\inf _{\hat{R}: f(\hat{R})=a} V^{\hat{R}}\left(a,\left(a_{0}, \kappa\left(a_{0}\right)\right)\right) .
$$

Further, individual rationality implies $\kappa\left(a_{0}\right) \leq 0$ (Lemma 1). Finally, Theorem 5 implies that if $(f, p)$ is a revenue maximizing mechanism then it must set $\kappa\left(a_{0}\right)=0$ and hence, for all $a \neq a_{0}$, we must have

$$
\kappa(a)=\inf _{\hat{R}: f(\hat{R})=a} V^{\hat{R}}\left(a,\left(a_{0}, 0\right)\right) .
$$

While finding the revenue maximizing mechanism in this general model may be hard, our results provide some properties of such a mechanism. For the simple (two alternative) 
setting of single object allocation to an agent, we use these findings to derive the revenue maximizing mechanism in Section 6.2.

Unfortunately, we do not know if this finding extends to a model with multiple agents - this is unlike Theorems 1, 2, and 3, which extend in a natural manner to a model with multiple agents. The main difficulty in extending Theorem 5 to a model with multiple agents is that when we change the allocation decision of one agent, it may require changes in allocation decision of other agents. As a result, even if we improve the revenue of one agent, we may end up decreasing revenue from other agents. We discuss these difficulties in more detail in the context of single object auction in Appendix B.

\section{Applications}

In this section, we give several applications of our results. We provide a brief preview of these applications.

In Section 6.1, we identify specific order respecting domains satisfying $\mathrm{TP}^{\epsilon}$ richness. By applying Theorems 1 and 2, we show how foundational results in quasilinear type spaces change when we consider type spaces which are arbitrarily small perturbations of quasilinear type space.

In Section 6.2, we apply Theorem 3 to derive a revenue maximizing mechanism for a seller selling a single object to a single agent.

In Section 6.3, we consider the problem of allocating a single object to $n$ agents using an anonymous and dominant strategy incentive compatible mechanism. We propose an extension of a Groves class of mechanisms to non-quasilinear preferences for this type space, and apply Theorem 3 to characterize it using anonymity, dominant strategy incentive compatibility, and a condition on the payment of losing agents.

In Section 6.4, we consider the problem of allocating $m$ objects to $n$ agents, each of whom can be assigned at most one object (unit demand preferences). Using our Theorem 1, we show that a naive generalization of the Vickrey-Clarke-Groves (VCG) mechanism may not be dominant strategy incentive compatible if preferences of agents are not quasilinear. We then provide a simple proof of dominant strategy incentive compatibility of a mechanism proposed by Demange and Gale (1985) in this problem.

\subsection{Perturbations of quasilinearity}

As we had observed earlier, Theorems 1 and 2 do not apply to quasilinear domains. We

show below that these results apply to domains that are "perturbations" of the quasilinear 
domain. This shows that even with arbitrarily small perturbation of quasilinear domain, we get revenue uniqueness instead of revenue equivalence. Further, the nature of monotonicity characterization changes even with a slight perturbation of quasilinearity.

We now define our notion of a perturbation. Fix a partial order $\succ$ over $A$. Let $a_{0} \in A$ be the minimal element with respect to $\succ$ - if $\succ$ is empty, then set $a_{0}$ equal to some alternative. Let $R$ and $R^{\prime}$ be any pair of preferences respecting $\succ$. Then, define the distance between $R$ and $R^{\prime}$ as

$$
d\left(R, R^{\prime}\right):=\sup _{t} \max _{a \neq a_{0}}\left|V^{R}\left(a,\left(a_{0}, t\right)\right)-V^{R^{\prime}}\left(a,\left(a_{0}, t\right)\right)\right| .
$$

Note that $d\left(R, R^{\prime}\right) \in \mathbb{R}_{+} \cup\{+\infty\}$. Further, $d\left(R, R^{\prime}\right)=d\left(R^{\prime}, R\right)$ and $d\left(R, R^{\prime}\right)=0$ if and only if $R=R^{\prime}$.

Let $\mathcal{D}^{\succ}$ be the set of all classical preferences respecting $\succ$. Let $\mathcal{R}^{\succ} \subseteq \mathcal{D}^{\succ}$ be some order respecting domain. For every $\epsilon>0$, define the $\epsilon$-perturbation of $\mathcal{R}^{\succ}$ as

$$
\mathcal{R}^{\succ, \epsilon}:=\left\{R \in \mathcal{D}^{\succ}: \exists R^{\prime} \in \mathcal{R}^{\succ} \text { with } d\left(R, R^{\prime}\right)<\epsilon .\right\}
$$

Informally, $\mathcal{R}^{\succ, \epsilon}$ contains all preferences in $\mathcal{D}^{\succ}$ which are within $\epsilon$ distance from some preference in $\mathcal{R}^{\succ}$. Notice that $\mathcal{R}^{\succ} \subset \mathcal{R}^{\succ, \epsilon}$.

We now describe a specific $\mathcal{R}^{\succ}$ such that $\mathcal{R}^{\succ, \epsilon}$ satisfies $\mathrm{TP}^{\epsilon}$-richness. This domain is derived from a problem with budget constrained agents but includes as a special case the quasilinear domain of preferences. Consider an agent who has a budget constraint $B$ and if the payment is less than $B$, then his preference is quasilinear, and is described by a valuation vector $\nu \in \mathbb{R}^{|A|}$ which respects a partial order $\succ$ over $A$. The interpretation of $B$ is standard: for any transfer above $B$, the agent borrows the extra amount with an interest rate $r \geq 0$. The interest rate $r$ is fixed for the problem, and it is possible that $r=0$ (in which case, it corresponds to the problem without any budget constraint, and the domain becomes the quasilinear domain). Formally, the utility of the agent for any alternative $a \in A$ and for transfer $t \leq B$ is

$$
\nu(a)-t
$$

and for transfer $t>B$ is

$$
\nu(a)-B-(t-B)(1+r) .
$$

Denote by $\mathcal{R}_{r}^{\succ}$ the set of all preferences representable by such $(\nu, B)$. As indicated above $\mathcal{R}_{0}^{\succ}$ is the set of quasilinear preferences respecting $\succ$. For an arbitrary $r \geq 0$ and $\epsilon>0$, the $\epsilon$-perturbation of the domain $\mathcal{R}_{r}^{\succ}$ is denoted by $\mathcal{R}_{r}^{\succ, \epsilon}$. Theorems 1 and 2 give us the following result. 
Proposition 1 Let $(f, p)$ be a mechanism defined over $\mathcal{R}_{r}^{\succ, \epsilon}$, where $r \geq 0$ and $\epsilon>0$. Then, the following statements are equivalent.

1. $(f, p)$ is incentive compatible.

2. $(f, p)$ is monotone and satisfies taxation principle.

Further, if $(f, p)$ and $\left(f, p^{\prime}\right)$ are two incentive compatible mechanisms defined on $\mathcal{R}_{r}^{\succ, \epsilon}$, then $p=p^{\prime}$.

The proof of Proposition 1 shows that $\mathcal{R}_{r}^{\succ}, \epsilon$ satisfies $\mathrm{TP}^{\epsilon}$-richness, and then the result follows from Theorems 1 and 2. Proposition 1 is showing that even with slight perturbation of quasilinearity, how the standard results from the quasilinear domain will change.

\subsection{Screening with non-quasilinearity}

The next two applications are for specific models of a private good provision. Consider a problem where a seller is selling a single indivisible object to a single buyer - the seller has zero utility for the object. Since the outcome is deterministic (either the buyer gets the object or not), the set of alternatives can be written as $A=\{0,1\}$, where 0 represents the alternative where the buyer does not get the object and 1 represents the alternative where he gets the object. The set of outcomes is $\mathcal{Z}:=A \times \mathbb{R}$, and the buyer (agent) has classical preferences over $\mathcal{Z}$. We consider the order respecting domain of preferences with respect to the order $1 \succ 0$, i.e., for any classical preference $R$ and any transfer $t$, we assume $(1, t) P(0, t)$.

For any classical preference $R$, we remind that the willingness to pay of buyer type $R$ at $t$ is

$$
W P(R, t):=V^{R}(1 ;(0, t))-t \quad \forall t \in \mathbb{R}
$$

So, $W P(R, t)$ is the amount that makes a buyer of type $R$ indifferent between not getting the object at transfer $t$ and getting the object at transfer $t+W P(R, t)$. Since $1 \succ 0$ and every $R$ respecting $\succ$, we have $W P(R, t)>0$ for all $R$ and for all $t$.

We now define the type space we work with in this model. We denote by $\mathcal{R}^{c}$ the set of all order respecting classical preferences in this model.

Definition 11 A type space $\mathcal{R} \subseteq \mathcal{R}^{c}$ satisfies strong WP-convexity if for every $t \in \mathbb{R}$, there exists $\beta_{t}>0$ such that

$$
\{W P(R, t): R \in \mathcal{R}\}=\left(0, \beta_{t}\right)
$$


Theorem 3 requires the type space to satisfy WP-convexity. Strong WP-convexity requires not only WP-convexity but also the fact that the interval of WPs at any $t$, must be of the form $\left(0, \beta_{t}\right)$, where $\beta_{t}>0$. Hence, the result in Theorem 3 can still be applied. In quasilinear domain, this richness will boil down to requiring that the valuation of the agent for the object lies in an open interval with lower support at zero. The definition above generalizes this richness to an arbitrary subset of classical type space.

Suppose $\mathcal{R}$ is the domain of preferences (type space). A mechanism $(f, p)$ in this case consists of an allocation rule $f: \mathcal{R} \rightarrow\{0,1\}$ and $p: \mathcal{R} \rightarrow \mathbb{R}$. In this section, we investigate the issue of expected revenue maximizing mechanism design. We search over all incentive compatible and individually rational mechanisms.

Definition 12 A mechanism $(f, p)$ is individually rational if for every $R \in \mathcal{R}$,

$$
(f(R), p(R)) R(0,0) \text {. }
$$

To compute expected payment from a mechanism, we need to have a prior over the types of the agents. By Theorem 3, a mechanism only needs information about the willingness to pay of the agent at one point (which will be determined as a part of the optimization exercise). Hence, the expected payment can be computed by using prior information on willingness to pay (WP). For every transfer $\tau \in \mathbb{R}$, we assume that WP values are drawn independently from a distribution. We denote it by $G_{\tau}$ at transfer $\tau$. Hence, $G_{\tau}(y)$ will denote the probability that the agent has a type $R$ such that $W P(R, \tau) \leq y$. We also assume that at every $\tau \in \mathbb{R}, G_{\tau}$ admits a positive density function, which we denote by $g_{\tau}$.

The expected revenue from a mechanism $M \equiv(f, p)$ is denoted as $\rho(M)$. This will be computed using the prior. If we are given a type space $\mathcal{R}$, we will denote by $\mathcal{M}(\mathcal{R})$ the set of all incentive compatible and individually rational mechanisms. We will say that a mechanism $M \in \mathcal{M}(\mathcal{R})$ is optimal in type space $\mathcal{R}$ if

$$
\rho(M) \geq \rho\left(M^{\prime}\right) \text { for all } M^{\prime} \in \mathcal{M}(\mathcal{R})
$$

We assume that $f$ is onto and apply Theorem 3 now - this is without loss of generality, since an optimal mechanism must be onto. By Theorem 3, every incentive compatible mechanism is described by $\tau^{0}$ and $f$. By Theorem 5, every optimal mechanism must have $\tau^{0}=0$. Hence, we only look at incentive compatible mechanisms such that $\tau^{0}=0$. Again using Theorem 3, the only unknown parameter is $\tau^{1}$. For a mechanism with $\tau^{0}=0$ and a fixed $\tau^{1}$, Theorem 3 implies that the expected revenue is

$$
\tau^{1}\left(1-G_{0}\left(\tau^{1}\right)\right)
$$


Standard first order condition implies that the optimal choice of $\tau^{1}$ must solve

$$
\tau^{1}=\frac{1-G_{0}\left(\tau^{1}\right)}{g_{0}\left(\tau^{1}\right)} .
$$

By strong WP-convexity, the possible values of $\tau^{1}$ lie in $\left(0, \beta_{0}\right)$. Choosing $\tau^{1} \rightarrow 0$ gives an expected revenue of $\rightarrow 0$. Similarly, choosing $\tau^{1} \rightarrow \beta_{0}$ gives an expected revenue of $\rightarrow 0$. Hence, the corner solutions are ruled out.

We impose the standard hazard rate condition.

Assumption MHR: $G_{0}$ satisfies monotone hazard rate (MHR) condition if $\frac{g_{0}(y)}{1-G_{0}(y)}$ is non-decreasing in $y$.

If $G_{0}$ satisfies MHR condition, then $y=\frac{1-G_{0}(y)}{g_{0}(y)}$ has a unique solution. Denote this unique solution as $r^{*}$.

Definition 13 A mechanism $(f, p)$ is a $r^{*}$-reserve price mechanism if for all $R \in \mathcal{R}$,

$$
\begin{aligned}
{\left[W P(R, 0)>r^{*}\right] } & \Rightarrow f(R)=1 \\
{\left[W P(R, 0)<r^{*}\right] } & \Rightarrow f(R)=0 \\
p(R) & =f(R) \cdot r^{*}
\end{aligned}
$$

In a $r^{*}$-reserve price mechanism, whenever the object is sold, the agent pays $r^{*}$. When the object is not sold, he pays 0 . The object is sold to the agent at price $r^{*}$ if his willingness to pay at 0 is larger than $r^{*}$. If the willingness to pay at 0 is less than $r^{*}$, then the object is not sold to the agent. Hence, an $r^{*}$-reserve price mechanism only requires WP information of agents at zero transfer. Our discussions in this section has shown that an $r^{*}$-reserve price mechanism is an optimal mechanism under the MHR assumption. We document this fact as a proposition.

Proposition 2 Suppose $\mathcal{R}$ satisfies strong WP-convexity and $G_{0}$ satisfies the $M H R$ condition. Then, an $r^{*}$-reserve price mechanism is an optimal mechanism in $\mathcal{M}(\mathcal{R})$.

Proposition 2 shows that the optimal mechanism for selling an indivisible object to one buyer is still very similar to the quasilinear domain. As we observed in remarks following Theorem 3, revenue equivalence may not hold in these models. In that sense, it is interesting that we are able to provide a solution of the optimal mechanism. Unfortunately, this is no longer true in the multiple agents case. We give a detailed analysis of the multiple agents case under additional assumptions in Appendix B, where we illustrate some of the difficulties in extending Proposition 2. 


\subsection{A characterization of anonymous auctions}

We now discuss an application of Theorem 3 for the many agents case. There is a single indivisible object to be allocated among $n$ agents. In our application, the designer is a social planner who is interested in allocating the object fairly. We use a minimal notion of fairness, which we call anonymity - outcomes of agents should not depend on their identities. We characterize the class of anonymous and dominant strategy incentive compatible mechanisms satisfying an additional property called the loser payment independence (LPI) - LPI requires that whenever an agent does not receive an object, his transfer amount is the same. This class of mechanisms can be thought to be a generalization of the class of Groves mechanisms for the quasilinear domains to this setting.

Formally, the set of agents is denoted by $N:=\{1, \ldots, n\}$. The type of each agent $i \in N$ is a classical preference $R$ over the consumption bundles $\mathcal{Z}=\{0,1\} \times \mathbb{R}$, and we also assume (as in previous subsection) that these classical preferences are order respecting with respect to $\succ$, where $1 \succ 0$. Let $\mathcal{R}$ denote any subset of such order respecting classical preferences.

An allocation rule is a map $f: \mathcal{R}^{n} \rightarrow N \cup\{0\}$, where choosing 0 indicates not giving the object to any of the agents. Notationally, at every type profile $\mathbf{R} \equiv\left(R_{1}, \ldots, R_{n}\right) \in \mathcal{R}^{n}$, we will write $f_{i}(\mathbf{R}) \in\{0,1\}$ to denote if agent $i$ gets the object at type profile $\mathbf{R}$. We use the standard notation $R_{-i}$ to denote a profile of types that does not include the type of agent $i$.

The payment rule of agent $i$ is a map $p_{i}: \mathcal{R}^{n} \rightarrow \mathbb{R}$. We will denote by $\mathbf{p} \equiv\left(p_{1}, \ldots, p_{n}\right)$ a collection of payment rules. A mechanism is a tuple $(f, \mathbf{p})$. The standard notion of dominant strategy incentive compatibility is defined as follows.

Definition 14 A mechanism $(f, \mathbf{p})$ is dominant strategy incentive compatible (DSIC) if for every $i \in N$, for every $R_{-i} \in \mathcal{R}^{n-1}$, and for every $R_{i}, R_{i}^{\prime} \in \mathcal{R}$, we have

$$
\left(f_{i}\left(R_{i}, R_{-i}\right), p_{i}\left(R_{i}, R_{-i}\right)\right) R_{i}\left(f_{i}\left(R_{i}^{\prime}, R_{-i}\right), p_{i}\left(R_{i}^{\prime}, R_{-i}\right)\right)
$$

We are interested in allocating the object using a fair mechanism. The notion of fairness we impose is the following.

Definition 15 A mechanism $(f, \mathbf{p})$ is anonymous if for every $i, j \in N$, for every $R_{-i j} \in$ $\mathcal{R}^{n-2}$, and for every $R_{i}, R_{j} \in \mathcal{R}$, we have

$$
\begin{aligned}
& \left(f_{i}\left(R_{i}, R_{j}, R_{-i j}\right), p_{i}\left(R_{i}, R_{j}, R_{-i j}\right)\right) I_{i}\left(f_{j}\left(R_{i}^{\prime}, R_{j}^{\prime}, R_{-i j}\right), p_{j}\left(R_{i}^{\prime}, R_{j}^{\prime}, R_{-i j}\right)\right) \\
& \left(f_{j}\left(R_{i}, R_{j}, R_{-i j}\right), p_{i}\left(R_{i}, R_{j}, R_{-i j}\right)\right) I_{j}\left(f_{i}\left(R_{i}^{\prime}, R_{j}^{\prime}, R_{-i j}\right), p_{j}\left(R_{i}^{\prime}, R_{j}^{\prime}, R_{-i j}\right)\right)
\end{aligned}
$$

where $R_{i}^{\prime}=R_{j}$ and $R_{j}^{\prime}=R_{i}$. 
Roughly, anonymity requires that identities of agents cannot be used to allocate and price the object. Specifically, it requires that if preferences of $i$ and $j$ are permuted (keeping preferences of other agents fixed), then $i$ should be indifferent between his consumption bundle at the old profile and $j$ 's consumption bundle at the new profile.

We now define a class of auctions, which we call the adjusted Vickrey auction with a variable reserve price. This is an extension of the Vickrey auction defined for this problem by Saitoh and Serizawa (2008). We can think of our mechanism as a class of Clarke-Groves mechanisms (Clarke, 1971; Groves, 1973) for allocating a single object with classical preferences. These mechanisms are defined by an anonymous but variable reserve price map:

$$
r: \mathcal{R}^{n-1} \rightarrow \mathbb{R}
$$

For every agent $i$, the reserve price of agent $i$ at a profile $\left(R_{i}, R_{-i}\right)$ is $r\left(R_{-i}\right)$. These mechanisms also specify $\tau^{0} \in \mathbb{R}$, which is the payment of any losing agent. To define the mechanism, we define the following notations. Given a reserve price map $r$, at every profile $\mathbf{R} \in \mathcal{R}^{n}$, define

$$
\begin{aligned}
W(\mathbf{R} ; r) & :=\left\{i \in N: W P\left(R_{i}, \tau^{0}\right) \geq \max \left(r\left(R_{-i}\right), \max _{j \neq i} W P\left(R_{j}, \tau^{0}\right)\right)\right\} \\
W^{*}(\mathbf{R} ; r) & :=\left\{i \in N: W P\left(R_{i}, \tau^{0}\right)>\max \left(r\left(R_{-i}\right), \max _{j \neq i} W P\left(R_{j}, \tau^{0}\right)\right)\right\} .
\end{aligned}
$$

Note that if $W^{*}(\mathbf{R} ; r)$ is non-empty, then it is a singleton. Further, $W(\mathbf{R} ; r)$ are the set of agents who have the highest willingness to pay $\left(\right.$ at $\tau^{0}$ ) and whose willingness to pay (at $\tau^{0}$ ) is higher than their respective reserve prices. Now, we describe the mechanism. The mechanism is a little complicated to describe because of tie-breaking issues. Informally, it fixes a reserve price for each agent for every type profile of other agents. At every type profile, it considers the set of agents who have the highest WP at $\tau^{0}$ and allocates the object to one of these agents if his willingness to pay is larger than the reserve price. Agents who do not win the object pay $\tau^{0}$ but if an agent wins the object then he pays $\tau^{0}$ plus the maximum of his reserve price and the highest willingness to pay of other agents. One way to interpret $\tau^{0}$ is that before the start of the auction, the auctioneer charges $\tau^{0}$ (if $\tau^{0}$ is negative, this is a subsidy but if $\tau^{0}$ is positive, this is an entry-fee) to all the agents, and then conducts a Vickrey auction with variable reserve price.

DEFINITION 16 The mechanism $\left(f^{*}, \mathbf{p}^{*}\right)$ is an adjusted Vickrey auction with a variable reserve price (AVVR) mechanism if there exists a real number $\tau^{0} \in \mathbb{R}$ and a map $r: \mathcal{R}^{n-1} \rightarrow \mathbb{R}$ such that at every type profile $\mathbf{R} \in \mathcal{R}^{n}$, we have

- if $W(\mathbf{R} ; r)=\emptyset$, then $f_{i}^{*}(\mathbf{R})=0$ for all $i \in N$, 
- if $i \in W^{*}(\mathbf{R} ; r)$, then $f_{i}^{*}(\mathbf{R})=1$, and

- if $f_{i}^{*}(\mathbf{R}) \neq 0$, then $i \in W(\mathbf{R} ; r)$,

and for every $i \in N$,

$$
p_{i}^{*}(\mathbf{R})=\tau^{0}+f_{i}^{*}(\mathbf{R}) \cdot \max \left(r\left(R_{-i}\right), \max _{j \neq i} W P\left(R_{j}, \tau^{0}\right)\right) .
$$

An example will clarify the AVVR mechanism. Consider $N=\{1,2\}$ and an AVVR mechanism with $\tau^{0}=0$. We define the reserve price map $r: \mathcal{R} \rightarrow \mathbb{R}$ as follows: for every $R \in \mathcal{R}$,

$$
r(R):=\frac{1}{W P(R, 0)} .
$$

The AVVR mechanism allocates the object to agent 1 if $W P\left(R_{1}, 0\right)>\max \left(W P\left(R_{2}, 0\right), \frac{1}{W P\left(R_{2}, 0\right)}\right)$ and to agent 2 if $W P\left(R_{2}, 0\right)>\max \left(W P\left(R_{1}, 0\right), \frac{1}{W P\left(R_{1}, 0\right)}\right)$. It does not allocate the object to any agent otherwise. The allocation is shown in Figure 6.

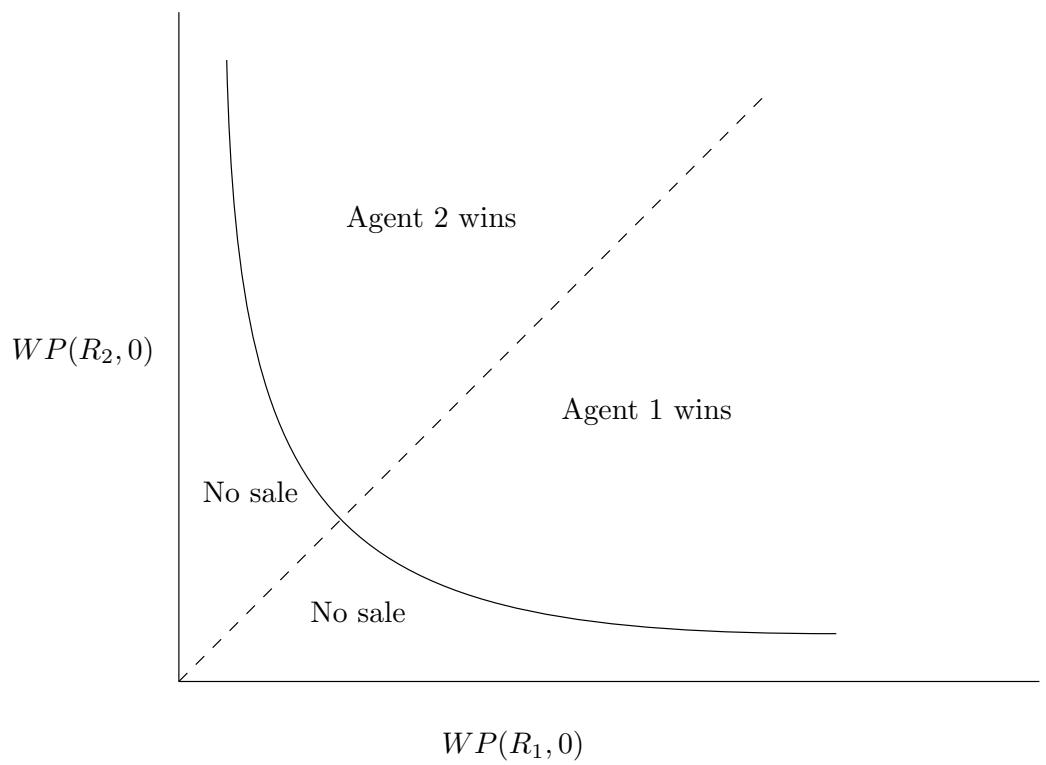

Figure 6: Allocation in an AVVR mechanism

The fact that AVVR mechanism uses personalized reserve prices distinguishes it from other mechanisms studied in this literature where mostly fixed reserve prices have been studied (Sakai, 2013a). ${ }^{4}$

\footnotetext{
${ }^{4}$ Yamashita (2015) discusses such personalized reserve price Vickrey auctions for quasilinear environment, but his objective is quite different.
} 
Payments in the AVVR mechanism is like a Vickrey auction with personalized reserve price - winning agent pays the maximum of his reserve price and the second highest willingness to pay (plus the $\tau^{0}$ amount). Usually, in an incentive compatible mechanism, $\tau^{0}$ could depend on the agent and the reports of other agents. But the AVVR mechanism uses simpler payments for losing agents. In general, we restrict our analysis below to such mechanisms.

Definition 17 A mechanism ( $f, \mathbf{p})$ satisfies loser payment independence (LPI) if there exists a real number $\tau^{0}$ such that for every $i \in N$ and for every $\mathbf{R} \in \mathcal{R}^{n}$ with $f_{i}(\mathbf{R})=0$ we have $p_{i}(\mathbf{R})=\tau^{0}$.

Note that LPI does not restrict $\tau^{0}$ to be zero or non-negative. Zero payment for losers or no-subsidy for losers is used as an axiom in the literature on auction design without quasilinearity - see for instance Saitoh and Serizawa (2008); Sakai (2013a); Morimoto and Serizawa (2015). LPI allows us some flexibility in setting the payment of the losers, and our AVVR mechanisms may not satisfy no-subsidy for losers but satisfy LPI.

We are now ready to state the characterization result for AVVR mechanism - it is proved in the Appendix using the $n$-agent extension of Theorem 3.

TheOREM 6 Suppose $\mathcal{R}$ is a classical type space satisfying strong WP-convexity. Let $(f, \mathbf{p})$ be a mechanism defined on $\mathcal{R}^{n}$. Then, the following statements are equivalent.

1. $(f, \mathbf{p})$ is DSIC, anonymous, and satisfies LPI.

2. $(f, \mathbf{p})$ is an $A V V R$ mechanism.

The proof in Theorem 6 constructs one reserve price map given a DSIC, anonymous mechanism satisfying LPI. But there can be other choices for the reserve price map also. Any such choice leads to an AVVR mechanism. Theorem 6 extends and strengthens a characterization of Vickrey auction in the quasilinear domain by Ashlagi and Serizawa (2012), who use anonymity, DSIC, individual rationality, and no-subsidy for losers as their axioms. In contrast, our characterization is valid for non-quasilinear domains and uses less restrictive LPI axiom in place of individual rationality and no-subsidy for losers.

\subsection{VCG may not work}

In Section 6.3, we showed that a natural extension of a class of Groves mechanisms continues to be DSIC in the single object allocation model. One of the reasons such an extension worked was that each agent had just two alternatives: either getting the object or not. In 
this section, we show that such an extension may not work if each agent had more than two alternatives.

How can we define an extension of the Vickrey-Clarke-Groves (VCG) mechanism in this setting if agents have classical preferences? We propose a naive extension. Let $N$ be a finite set of agents. Let $A$ be a finite set of alternatives that any agent $i \in N$ can be allocated. For simplicity of exposition, we assume existence of an alternative $a_{0}$ and a partial order $\succ$ such that $a \succ a_{0}$ for all $a \in A$. The preferences we consider here will respect such a partial order $\succ$.

Let $\mathcal{R}^{\succ}$ denote the set of classical preferences over $A \times \mathbb{R}$ respecting $\succ$. A mechanism will allocate a collection of alternatives $\left(a_{1}, \ldots, a_{n}\right)$ at each profile of preferences, where $a_{i}$ is the alternative allocated to agent $i$. Notice that there may be restrictions on such allocations for instance, in the public good problems, we may impose $a_{i}=a_{j}$ for all $i, j \in N$; for private good allocation problems where no agent can be assigned the same alternative except $a_{0}$ (e.g., the unit-demand multi-object allocation problem discussed in Section 6.4.1), we may impose $a_{i} \neq a_{j}$ for all $i, j \in N$ if $a_{i}, a_{j} \neq a_{0}$. Let $\mathcal{A} \subseteq A^{n}$ be the set of all feasible allocations of alternatives to agents.

A mechanism is a pair $(f, \mathbf{p})$, where the allocation rule $f$ is a map $f:\left(\mathcal{R}^{\succ}\right)^{n} \rightarrow \mathcal{A}$ and $\mathbf{p} \equiv\left(p_{1}, \ldots, p_{n}\right)$ is a collection of payment rules, where $p_{i}:\left(\mathcal{R}^{\succ}\right)^{n} \rightarrow \mathbb{R}$ is the payment rule for agent $i$. At any preference profile $\mathbf{R}, f_{i}(\mathbf{R})$ is the alternative assigned to agent $i$ and $p_{i}(\mathbf{R})$ is the payment of agent $i$. As usual, a mechanism $(f, \mathbf{p})$ is dominant strategy incentive compatible (DSIC) if for all $i \in N$, for all $R_{-i}$, and for all $R_{i}, R_{i}^{\prime}$, we have

$$
\left(f_{i}\left(R_{i}, R_{-i}\right), p_{i}\left(R_{i}, R_{-i}\right)\right) R_{i}\left(f_{i}\left(R_{i}^{\prime}, R_{-i}\right), p_{i}\left(R_{i}^{\prime}, R_{-i}\right)\right) .
$$

Note that with these $n$-agent notations, our definitions of monotonicity and the results in Theorems 1 and 2 extend in a straightforward manner.

Consider a profile of preferences $\mathbf{R} \equiv\left(R_{1}, \ldots, R_{n}\right)$. For each agent $i \in N$, we consider the valuation vector $\left\{V^{R_{i}}\left(a,\left(a_{0}, 0\right)\right)\right\}_{a \in A}$. We denote this vector as $v_{i} \in \mathbb{R}_{+}^{|A|}$, where we note that $v_{i}\left(a_{0}\right)=0$. If $R_{i}$ is quasilinear, $v_{i}$ will exactly correspond to the valuation of agent $i$ for alternatives in $A$. Denote the VCG payment of agent $i$ with quasilinear preference profile corresponding to $\mathbf{v} \equiv\left(v_{1}, \ldots, v_{n}\right)$ as $p_{i}^{v c g}(\mathbf{v})$ and his alternative allocation as $f_{i}^{v c g}(\mathbf{v}) \in A .^{5}$ The generalized $V C G$ mechanism $\left(f, \mathbf{p} \equiv\left(p_{1}, \ldots, p_{n}\right)\right)$ for classical preference profile $\mathbf{R}$ is now defined as: for all $i \in N$,

$$
f_{i}(\mathbf{R}):=f_{i}^{v c g}(\mathbf{v}) ; \quad p_{i}(\mathbf{R}):=p_{i}^{v c g}(\mathbf{v}) .
$$

\footnotetext{
${ }^{5}$ Informally, the VCG mechanism allocates objects such that the total valuation of agents is maximized. The payment of each agent is his externality to other agents - see Vohra (2011) or Borgers et al. (2015) for a formal definition.
} 
When there are two alternatives $A=\left\{a_{0}, a\right\}$, the generalized VCG mechanism is DSIC. Indeed Saitoh and Serizawa (2008) investigate the problem of allocating multiple homogeneous objects where each agent can consume at most one unit, and show that the generalized VCG is DSIC. In their model, every agent is either allocated an object $(a)$ or not allocated any object $\left(a_{0}\right)$. For the public good provision problem (where, you either provide the public good $(a)$ or not $\left.\left(a_{0}\right)\right)$, Hashimoto and Saitoh (2010) show that the generalized VCG mechanism is DSIC. It is not difficult to verify that for $|A|=2$, the generalized VCG mechanism satisfies WP-monotonicity and the payment formula of Theorem 3 (take $\tau^{0}=0$ for each agent). Hence, by Theorem 3, it is DSIC. ${ }^{6}$

We show below that this feature of the generalized VCG mechanism breaks down if $|A|>2$. In particular, we consider a particular private good allocation problem, and show that the generalized VCG mechanism violates monotonicity in that problem.

\subsubsection{Unit demand multi-object allocation}

In this section, we consider a private good allocation problem, where a designer has $m$ objects to allocate, denoted by $M:=\{a, b, c, \ldots\}$. There are $n$ agents (buyers), denoted by $N:=\{1, \ldots, n\}$. Each agent can receive at most one object (unit-demand preference). Let $A \equiv M \cup\left\{a_{0}\right\}$, where $a_{0}$ is the null object, which is assigned to any agent who does not receive any object in $M$. Each agent $i$ has classical preference $R_{i}$ over $A \times \mathbb{R}$ respecting the partial order $\succ: a \succ a_{0}$ for all $a \in M .^{7}$

We first show that in this model, the generalized VCG may not be DSIC by showing that it violates monotonicity. For this, consider $M=\{a, b\}$ and three agents $N=\{1,2,3\}$. For this, we consider two classical preference profiles: $\left(R_{1}, R_{2}, R_{3}\right)$ and $\left(\hat{R}_{1}, R_{2}, R_{3}\right)$. The corresponding valuations for each alternative at $\left(a_{0}, 0\right)$ are shown in Table 1.

It is not difficult to see that the total valuation-maximizing allocation at profile $\left(R_{1}, R_{2}, R_{3}\right)$ assigns agent 1 object $b$, agent 2 object $a$ and agent 3 object $a_{0}$. Hence,

$$
f_{1}\left(R_{1}, R_{2}, R_{3}\right)=b, f_{2}\left(R_{1}, R_{2}, R_{3}\right)=a, f_{3}\left(R_{1}, R_{2}, R_{3}\right)=a_{0}
$$

Similarly, for profile $\left(\hat{R}_{1}, R_{2}, R_{3}\right)$, we have

$$
f_{1}\left(\hat{R}_{1}, R_{2}, R_{3}\right)=a, f_{2}\left(R_{1}, R_{2}, R_{3}\right)=a_{0}, f_{3}\left(R_{1}, R_{2}, R_{3}\right)=b .
$$

\footnotetext{
${ }^{6}$ It is enough to show that the generalized VCG mechanism is DSIC in the domain which includes all classical preferences, and this type space satisfies WP-convexity assumption in Theorem 3.

${ }^{7}$ This is a well-studied problem in quasilinear domain - applications include allocation of public housing in cities, allocation of advertisement slots on a search page of a keyword and so on. We refer to Kazumura et al. (2017) for more applications of this problem.
} 


\begin{tabular}{|c||c|c|c|}
\hline & $a_{0}$ & $a$ & $b$ \\
\hline$V^{R_{1}}\left(\cdot,\left(a_{0}, 0\right)\right)$ & 0 & 5 & 8 \\
\hline$V^{R_{2}}\left(\cdot,\left(a_{0}, 0\right)\right)$ & 0 & 4 & 5 \\
\hline$V^{R_{3}}\left(\cdot,\left(a_{0}, 0\right)\right)$ & 0 & 3 & 6 \\
\hline \hline$V^{\hat{R}_{1}}\left(\cdot,\left(a_{0}, 0\right)\right)$ & 0 & 5 & 4 \\
\hline
\end{tabular}

Table 1: Valuations at $\left(a_{0}, 0\right)$

We only compute the payments of agent 1 , which can be done by computing his externalities on other agents. We only do it at profile $\left(\hat{R}_{1}, R_{2}, R_{3}\right)$. Routine calculations show: $p_{1}\left(\hat{R}_{1}, R_{2}, R_{3}\right)=4$. Hence, agent 1 is allocated $(a, 4)$ at preference profile $\left(\hat{R}_{1}, R_{2}, R_{3}\right)$ and he gets object $b$ at preference profile $\left(R_{1}, R_{2}, R_{3}\right)$. We now argue that $R_{1}$ and $\hat{R}_{1}$ can be chosen in a way such that monotonicity is violated.

So far, we have not used any other feature of the classical preferences except their valuations at $\left(a_{0}, 0\right)$. Hence, it is possible to choose $R_{1}$ and $\hat{R}_{1}$ such that $V^{R_{1}}(b,(a, 4))<$ $V^{\hat{R}_{1}}(b,(a, 4))$ - this is shown in Figure 7 . This is a violation of monotonicity. Thus, by Theorem 1, the generalized VCG mechanism violates DSIC.

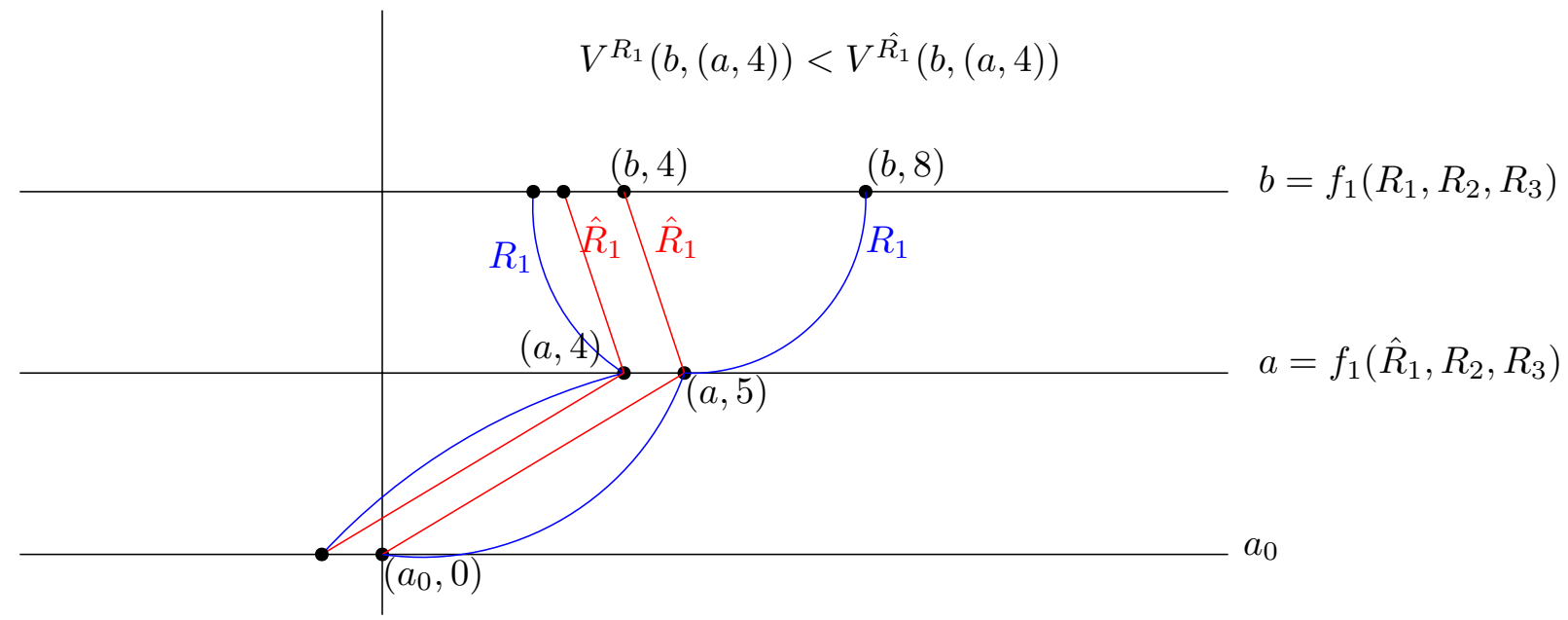

Figure 7: Violation of monotonicity by the generalized VCG mechanism

We emphasize that the generalized VCG mechanism satisfies the taxation principle in all problems. Hence, monotonicity is a key condition for the generalized VCG mechanism to satisfy incentive compatibility. In other words, the generalized VCG mechanism is DSIC in a problem if and only if it satisfies monotonicity in that problem. 


\subsubsection{The minimum Walrasian equilibrium price mechanism}

We now turn our attention to a mechanism proposed by Demange and Gale (1985) in the unitdemand multi-object allocation problem discussed in the previous section. This mechanism is DSIC. It also inherits all the properties of the VCG mechanism from the quasilinear preference domain - for instance, it is Pareto efficient and individually rational.

To define this mechanism, we will need some extra notations. A price vector $\pi \in \mathbb{R}_{+}^{|A|}$ defines a price for every object with $\pi_{a_{0}}=0$. At any price vector $\pi$, let $D\left(R_{i}, \pi\right):=\{a \in A$ : $\left.\left(a, \pi_{a}\right) R_{i}\left(b, \pi_{b}\right) \forall b \in A\right\}$ denote the demand set of agent $i$ with preference $R_{i}$.

An object allocation is an $n$-tuple $\left(a_{1}, \ldots, a_{n}\right) \in A^{n}$ such that no real (non-null) object is assigned to two agents, i.e., $a_{i} \neq a_{j}$ for all $i, j$ with $a_{i}, a_{j} \neq 0$.

Definition 18 An object allocation $\left(a_{1}, \ldots, a_{n}\right)$ and a price vector $\pi$ is a Walrasian equilibrium at a preference profile $\mathbf{R} \equiv\left(R_{1}, \ldots, R_{n}\right)$ if

1. $a_{i} \in D\left(R_{i}, \pi\right)$ for all $i \in N$ and

2. for all $a \in M$ with $a_{i} \neq a$ for all $i \in N$, we have $\pi_{a}=0$.

We refer to $\pi$ defined above as a Walrasian equilibrium price vector.

A Walrasian equilibrium price vector $\pi$ is a minimum Walrasian equilibrium price vector at preference profile $\mathbf{R}$ if for every Walrasian equilibrium price vector $\pi^{\prime}$ at $\mathbf{R}$, we have $\pi_{a} \leq \pi_{a}^{\prime}$ for all $a \in A$. Demange and Gale (1985) prove that a Walrasian equilibrium exists at every preference profile $\mathbf{R}$, and the set of Walrasian equilibrium price vectors forms a compact lattice. We denote the minimum Walrasian equilibrium price vector at $\mathbf{R}$ as $\pi^{\min }(\mathbf{R})$.

Although the minimum Walrasian equilibrium price vector is unique at every preference profile, there may be multiple supporting object allocation - all these object allocations must be indifferent to all the agents. To handle this multiplicity problem, we introduce some notation. Let $Z^{\min }(\mathbf{R})$ denote the set of all allocations at a minimum Walrasian equilibrium at preference profile $\mathbf{R}$. Note that if $\left(\left(a_{1}, \ldots, a_{n}\right), \pi\right) \in Z^{\min }(\mathbf{R})$ then $\pi=\pi^{\min }(\mathbf{R})$. The minimum Walrasian equilibrium price mechanism is defined as follows.

Definition 19 A mechanism $(f, \mathbf{p})$ is a minimum Walrasian equilibrium price (MWEP) mechanism if $(f(\mathbf{R}), \mathbf{p}(\mathbf{R})) \in Z^{\min }(\mathbf{R}) \forall \mathbf{R}$.

We reprove the following result.

Proposition 3 (Demange and Gale (1985)) Every MWEP mechanism is DSIC. 
The proof is provided in Appendix A. The original proof of Proposition 3 in Demange and Gale (1985) is quite involved. A simpler proof is provided in Morimoto and Serizawa (2015). Their proof uses a characterization of MWEP in terms of demand sets of agents. We use the necessity part of this characterization along with our Theorem 1 to establish an even simpler proof of Proposition 3.

The unit demand multi-object allocation problem is the only known problem in the nonquasilinear preference environment where the generalized VCG mechanism does not work and still a Pareto efficient and DSIC mechanism exists. A natural future research agenda will be to investigate problems where we can find Pareto efficient and DSIC mechanisms - in the literature review (in Section 7), we report various existing impossibility results in that direction. We hope that our Theorem 1 will be useful to map this frontier of possibility and impossibility.

\section{Related literature}

The literature on mechanism design with quasilinearity is long, and almost impossible to describe exhaustively. We discussed some relevant papers in detail in Section 4.3, but refer to the reader to two excellent books on this topic (Vohra, 2011; Borgers et al., 2015). After Myerson (1981), a long literature has focused on extending his monotonicity and revenue equivalence characterizations to various models with multidimensional type space, where more than two alternatives are allocated and agents have values for each of those alternatives. Because of quasilinearity, the allocation rule and payment rule of a mechanism appear in separable form in the utility function of the agent. This allows one to provide separate characterization of implementable allocation rules (i.e., allocation rules for which payment rules can be found to make the tuple incentive compatible) and the class of payment rules that implement an implementable allocation rule. The former is characterized by a simple monotonicity property - results in that spirit appear in Jehiel et al. (1999); Bikhchandani et al. (2006); Saks and Yu (2005); Ashlagi et al. (2010); Cuff et al. (2012); Muller et al. (2007); Mishra and Roy (2013); Mishra et al. (2014); Carbajal and Müller (2015). ${ }^{8}$ The latter is characterized by a revenue equivalence (or envelope theorem) formula - results in that spirit are in Krishna and Maenner (2001); Milgrom and Segal (2002); Chung and Olszewski (2007); Heydenreich et al. (2009). These results exploit the geometric structure induced by quasilinearity, and make use of convex analysis machinery (Rockafellar, 1970). A paper by

\footnotetext{
8 These papers (a) extend Myerson's monotonicity condition to multidimensional environment and (b) simplify a complicated monotonicity characterization of Rochet (1987).
} 
Carbajal and Ely (2013) establishes that revenue equivalence may not hold in some problems even with quasilinearity - this is due to non-convex and non-differentiable nature of valuation function. They further show that in specific problems where revenue equivalence fails, one can still do revenue maximization using revenue inequalities.

In the absence of quasilinearity, it is not possible to provide separate characterization of monotonicity and revenue equivalence since the allocation and payment are no longer separable. Our monotonicity condition is indeed a condition on the mechanism, but reduces to the monotonicity condition on allocation rule used in quasilinear type spaces. Unlike the quasilinear type space, we find revenue uniqueness of incentive compatible mechanisms in our benchmark rich classical type space. Our revenue uniqueness is driven by the nonquasilinearity of preferences and the underlying richness of the type space that we assume. Unlike the literature, we do not (and cannot) rely on convex analysis techniques. A crucial difference of our model and that of the literature on quasilinear preferences is that we assume no functional form of utility functions. Our restriction on type spaces are on the primitives of the model, which is the classical preferences.

There is a short but important literature on mechanism design with non-quasilinear preferences. The closest paper to ours is Kos and Messner (2013). They derive a necessary condition for incentive compatibility in a model with non-quasilinearity. Their condition is a generalization of the cycle monotonicity condition in Rochet (1987) for quasilinear preferences. They show that their condition is not sufficient for incentive compatibility. In contrast, our monotonicity condition is significantly weaker than their condition and is a necessary and sufficient condition for incentive compatibility along with the taxation principle. However, we focus on deterministic mechanisms assuming some richness in type space, and Kos and Messner (2013) do not make such assumptions.

Baisa (2017) considers the single object auction model and allows for randomization in a model with non-quasilinear preferences. He introduces a novel mechanism in his setting and studies its optimality properties (in terms of revenue maximization). Garratt and Pycia (2016) study the bilateral trading model with non-quasilinear preferences and allows for randomization. Further, their solution concept is Bayesian incentive compatibility. Their main finding is that for generic set of non-quasilinear preferences, the Myerson-Satterthwaite impossibility result (Myerson and Satterthwaite, 1983) on bilateral trading disappears. Unlike both these papers, we do not consider randomization and our solution concept is different from them. More importantly, we have a more general model of non-quasilinear preferences, albeit with deterministic mechanisms, which covers many problems of interest. In a recent paper, Noldeke and Samuelson (2015) analyze principal-agent problem and matching prob- 
lem with non-quasilinear preferences. They establish a certain implementation duality using abstract convex analysis techniques, which allows them to extend results from quasilinear type spaces. Our results are specific to deterministic mechanisms, which is not assumed in Noldeke and Samuelson (2015). The objectives of our paper and that of Noldeke and Samuelson (2015) are quite different, and hence, the results are quite different.

There is a literature on axiomatic treatment of mechanisms in models without quasilinearity. This literature identifies specific mechanisms in different problems with classical preferences and axiomatically characterizes these mechanisms. The central axiom in this literature is dominant strategy incentive compatibility, but it is often accompanied by Pareto efficiency, individual rationality, and other axioms. As discussed earlier, a seminal paper in this literature is Demange and Gale (1985), who consider the unit demand multi-object allocation problem without quasilinearity and define the MPWE mechanism. They show that the MPWE mechanism is stable and dominant strategy incentive compatible. Morimoto and Serizawa (2015) characterize the MPWE mechanism using Pareto efficiency, individual rationality, incentive compatibility, and other axioms on payments - see an extension of this characterization in a smaller type space in Zhou and Serizawa (2016). These characterizations require a type space that is rich in a strong sense - often, a type space that includes all classical preferences. Saitoh and Serizawa (2008); Sakai (2008) define the generalization of Vickrey auction in the single object auction model with non-quasilinear preferences. They characterize the Vickrey auction in a rich non-quasilinear type space using incentive compatibility, Pareto efficiency, individual rationality, and no-subsidy for losers - see also Ashlagi and Serizawa (2012) for an analogous characterization of Vickrey auction in the quasilinear domain. Sakai (2013b) and Adachi (2014) characterize the same generalized Vickrey auction mechanism using incentive compatibility, continuity, and some fairness axioms. Sakai (2013a) considers an extension of this generalized Vickrey auction with reserve price, and characterizes it using incentive compatibility, weak Pareto efficiency, and zero-payment for losers. The mechanism we propose for allocating an object is more general than the auction in Sakai (2013a) since it has personalized reserve prices for every agent which vary with preferences of other agents. Hashimoto and Saitoh (2010) defines the generalization of the Pivotal mechanism in the single public good provision model with non-quasilinear preferences and characterize it using Pareto efficiency, incentive compatibility, and an equity axiom.

When the set of preferences include all or a very rich class of non-quasilinear preferences, impossibility results exist in various problems. Several authors have shown that no incentive compatible and Pareto efficient mechanism exists that satisfy other mild axioms - Kazumura and Serizawa (2016) show this for multi-object auction problems where agents can 
be allocated more than one object; Baisa (2016) shows this for homogeneous object allocation problems; Ma et al. (2016) shows a similar result for public good provision problem and Dobzinski et al. (2012); Lavi and May (2012) show similar results for hard budget-constrained auction of a single object. Pareto efficiency along with other axioms play a crucial role in such impossibility results. Unlike these papers, we do not impose any extra condition besides incentive compatibility. Moreover, we also cover domains where possibility exists with Pareto efficiency and other axioms.

Summarizing, the main differences between this literature and our paper is that (a) we have results for a very general class of problems whereas each paper in this literature focuses on a particular mechanism defined on a specific problem; (b) our focus on axiomatic characterization of a class of mechanisms for single object allocation illustrates the use of a particular general result - in particular, our AVVR class of mechanisms are more general than any specific mechanism discussed in the literature.

There is a literature in auction theory and algorithmic game theory on single object auctions with budget-constrained bidders - see Che and Gale (2000); Pai and Vohra (2014); Ashlagi et al. (2010); Lavi and May (2012). Carbajal and Mualem (2017) considers the budget-constrained single object auction problem. They extend Rochet's cycle monotonicity (Rochet, 1987) characterization to their problem under additional conditions, and find conditions under which revenue equivalence holds. The budget-constraint in these papers introduces a particular form of non-quasilinearity in preferences of agents. Further, the budget-constraint in these models is hard, i.e., the utility from any payment above the budget is minus infinity. This assumption is not satisfied by the preferences considered in our model. Hence, we cannot apply our results to these models directly. However, if we consider soft budget-constraints (where agents can borrow from banks at an interest rate), then our results on the two-alternatives case will apply to some of these models.

In a companion paper (Kazumura et al., 2017), we investigate the question of revenue maximizing auction by a seller who is selling multiple objects to agents who can buy at most one object. The minimum Walrasian equilibrium price mechanism is shown to be revenue-optimal in a class of mechanisms satisfying dominant strategy incentive compatibility, individual rationality and some other reasonable axioms. The technique used in that paper is quite different from the current paper. Moreover, the problem studied in that paper is very specific and involves axioms other than incentive compatibility and individual rationality. 


\section{Conclusion}

We have presented a systematic analysis of the structure of incentive compatible mechanisms when agents have non-quasilinear preferences. Our characterization results give novel insights into the complex nature of incentive constraints in this model. It also explores the tractability of performing optimization with non-quasilinear preferences. Our results are detail-free in the sense that they do not assume any particular functional form of non-quasilinear preferences. We apply our results to some of the models where similar results with quasilinear preferences have been successfully applied. While we were successful in some of them, we only have partial answers in others. This shows the delicate nature of incentive compatible mechanisms as we move from quasilinear preferences to general classical preferences.

A troubling feature of mechanisms in non-quasilinear type spaces is the informational requirement - preferences over infinite number of consumption bundles have to be elicited. Mechanisms that only elicit finite amount of information must be studied and its performance must be compared with a general mechanism. This will be crucial to apply mechanism design theory to type spaces without quasilinearity. We leave this important issue as an area of future research. Another area of future research will be to find specific models of non-quasilinear type spaces and apply our results in such models. 


\section{Appendix A: Omitted proofs}

\section{Proofs of Section 4 and Section 5}

\section{Proof of Theorem 1}

Proof: Suppose $(f, p)$ is incentive compatible. Consider any $R, R^{\prime}$ with $f(R)=f\left(R^{\prime}\right)=a$. By incentive compatibility

$$
(a, p(R)) R\left(a, p\left(R^{\prime}\right)\right) \text { and }\left(a, p\left(R^{\prime}\right)\right) R^{\prime}(a, p(R)) \text {. }
$$

Money monotonicity of classical preferences implies that $p(R)=p\left(R^{\prime}\right)$. Hence, define for every $a \in A, \kappa(a):=p(R)$ for some $R \in \mathcal{R}$ with $f(R)=a$. Hence, taxation principle holds.

Pick $R, R^{\prime} \in \mathcal{R}$ such that $f(R)=a, f\left(R^{\prime}\right)=b$. By incentive compatibility

$$
(a, \kappa(a)) R(b, \kappa(b)) I\left(a, V^{R}(a,(b, \kappa(b)))\right) .
$$

Hence, $\kappa(a) \leq V^{R}(a,(b, \kappa(b)))$. But incentive compatibility also implies

$$
\left(a, V^{R^{\prime}}(a,(b, \kappa(b)))\right) I^{\prime}(b, \kappa(b)) R^{\prime}(a, \kappa(a)) .
$$

Hence, $\kappa(a) \geq V^{R^{\prime}}(a,(b, \kappa(b)))$. Combining these two observations, we get

$$
V^{R}(a,(b, \kappa(b))) \geq V^{R^{\prime}}(a,(b, \kappa(b))) .
$$

Hence, $(f, p)$ is monotone.

We now establish the converse implication. Suppose $(f, p)$ is monotone and there exists $\kappa: A \rightarrow \mathbb{R}$ such that for every $R, p(R)=\kappa(f(R))$.

Assume for contradiction that there is a preference $R$ such that $f(R)=a$ and another preference $R^{\prime}$ such that $f\left(R^{\prime}\right)=b \neq a$ but $(b, \kappa(b)) P(a, \kappa(a))$. This implies that $\kappa(a)>$ $V^{R}(a,(b, \kappa(b)))$. By monotonicity,

$$
V^{R}(a,(b, \kappa(b))) \geq V^{R^{\prime}}(a,(b, \kappa(b))) \text { and } V^{R^{\prime}}(b,(a, \kappa(a))) \geq V^{R}(b,(a, \kappa(a))) .
$$

Since $\kappa(a)>V^{R}(a,(b, \kappa(b)))$, we get $\kappa(b)<V^{R}(b,(a, \kappa(a)))$.

Choose $\epsilon^{\prime}>0$ but arbitrarily close to zero, and let $v \in \mathbb{R}^{|A \backslash\{a\}|}$ be such that for each $c \neq a$,

$$
v_{c}= \begin{cases}\kappa(b) & \text { if } c=b \\ \min \left(V^{R}(c,(b, \kappa(b))), V^{R^{\prime}}(c,(b, \kappa(b)))\right)-\epsilon^{\prime} & \text { if } c \neq b\end{cases}
$$


Define the set of preferences $\mathcal{R}^{*}$ as follows:

$$
\mathcal{R}^{*}:=\left\{\hat{R} \in \mathcal{R}^{\succ}: V^{\hat{R}}(c,(b, \kappa(b)))=v_{c} \forall c \notin\{a, b\}\right\} .
$$

Now, we do the proof in many steps.

STEP 1. We first show that $\mathcal{R}^{*}$ is non-empty. To do so, consider the following vector $v^{\prime \prime} \in \mathbb{R}^{|A|}$ as follows:

$$
v_{c}^{\prime \prime}= \begin{cases}v_{c} & \text { if } c \neq a \\ \min \left(V^{R}(a,(b, \kappa(b))), V^{R^{\prime}}(a,(b, \kappa(b)))\right)-\epsilon^{\prime} & \text { if } c=a .\end{cases}
$$

Next, we show that for sufficiently small $\epsilon^{\prime}>0, v^{\prime \prime}$ respects $\succ$. We consider various cases.

CAse 1. Suppose $b \succ c$, where $c \neq b$. Now,

$$
v_{b}^{\prime \prime}=\kappa(b)>\min \left(V^{R}(c,(b, \kappa(b))), V^{R^{\prime}}(c,(b, \kappa(b)))\right)>v_{c}^{\prime \prime},
$$

where the first inequality comes from the fact that $R$ and $R^{\prime}$ respect $\succ$. If $c \succ b$, then

$$
v_{c}^{\prime \prime}=\min \left(V^{R}(c,(b, \kappa(b))), V^{R^{\prime}}(c,(b, \kappa(b)))\right)-\epsilon^{\prime}>\kappa(b)=v_{b}^{\prime \prime}
$$

where the inequality follows because $R$ and $R^{\prime}$ respect $\succ$ and $\epsilon^{\prime}$ is sufficiently close to zero.

Case 2. Suppose $c \succ d$, where $c, d \neq b$. Then,

$v_{c}^{\prime \prime}=\min \left(V^{R}(c,(b, \kappa(b))), V^{R^{\prime}}(c,(b, \kappa(b)))\right)-\epsilon^{\prime}>\min \left(V^{R}(d,(b, \kappa(b))), V^{R^{\prime}}(d,(b, \kappa(b)))\right)-\epsilon^{\prime}=v_{d}^{\prime \prime}$,

where the strict inequality follows from the fact that $R$ and $R^{\prime}$ respect $\succ$.

This completes all the cases, and establishes the fact that $v^{\prime \prime}$ respects $\succ$. By $\mathrm{TP}^{\epsilon}$ richness, there is $\hat{R}^{\prime} \in \mathcal{R}^{\succ}$ such that $v^{\prime \prime} \in \mathcal{I}\left(\hat{R}^{\prime}\right)$. ${ }^{9}$ Since $v_{c}^{\prime \prime}=v_{c}$ for all $c \neq a$, we get that $\mathcal{R}^{*} \neq \emptyset$.

STEP 2. In this step, we show that $f(\hat{R}) \in\{a, b\}$ for all $\hat{R} \in \mathcal{R}^{*}$. To see this, suppose $f(\hat{R})=c \notin\{a, b\}$. By $f\left(R^{\prime}\right)=b$ and monotonicity, we get that $V^{\hat{R}}(c,(b, \kappa(b)))=v_{c} \geq$ $V^{R^{\prime}}(c,(b, \kappa(b)))$, which contradicts the definition of $v$.

\footnotetext{
${ }^{9} \mathrm{TP}^{\epsilon}$ richness ensures existence of a preference which has two pairwise consistent and $\epsilon$-parallel as indifference vectors. If we are only given one such indifference vector, we can choose another indifference vector trivially such that both are pairwise consistent and and $\epsilon$-parallel. Then, we can apply $\mathrm{TP}^{\epsilon}$ richness trivially.
} 
STEP 3. In this step, we construct two vectors which are pairwise consistent for $\succ$ and $\epsilon$-parallel, where $\epsilon>0$ is as defined in the statement of the theorem. To do so, we first define

$$
\eta:=\sup _{\hat{R} \in \mathcal{R}^{*}: f(\hat{R})=b} V^{\hat{R}}(a,(b, \kappa(b))) .
$$

To see that $\eta$ is well defined and finite, consider the preference $\hat{R}^{\prime} \in \mathcal{R}^{*}$ constructed at the end of Step 1. By Step 2, $f\left(\hat{R}^{\prime}\right) \in\{a, b\}$. If $f\left(\hat{R}^{\prime}\right)=a$, then monotonicity implies that

$$
v_{a}^{\prime \prime}=V^{\hat{R}^{\prime}}(a,(b, \kappa(b))) \geq V^{R^{\prime}}(a,(b, \kappa(b))) .
$$

But this contradicts the definition of $v_{a}^{\prime \prime}$. Hence, $f\left(\hat{R}^{\prime}\right)=b$. Finally, for every $\hat{R} \in \mathcal{R}^{*}$ with $f(\hat{R})=b$, monotonicity implies that $V^{R}(a,(b, \kappa(b))) \geq V^{\hat{R}}(a,(b, \kappa(b)))$. Hence, $\eta \leq$ $V^{R}(a,(b, \kappa(b)))$. This establishes that $\eta$ is a finite real number. Note that

$$
\eta \leq V^{R}(a,(b, \kappa(b)))<\kappa(a)
$$

Now, since $\mathcal{R}^{\succ}$ is $\mathrm{TP}^{\epsilon}$ rich, for every pair of $\epsilon$-parallel $v^{\prime}, v^{\prime \prime} \in \mathbb{R}^{|A|}$ which are pairwise consistent for $\succ$, there is a preference $R^{\prime \prime}$ such that $v^{\prime}, v^{\prime \prime} \in \mathcal{I}\left(R^{\prime \prime}\right)$. Choose, $\epsilon^{*} \in\left(0, \frac{\epsilon}{3}\right)$ and arbitrarily close to zero. Let $v^{*}$ be such that for each $c \in A$,

$$
v_{c}^{*}= \begin{cases}\eta-\epsilon^{*} & \text { if } c=a \\ v_{c} & \text { otherwise. }\end{cases}
$$

Since $\eta$ is well defined, there exists $\hat{R} \in \mathcal{R}^{*}$ such that $V^{\hat{R}}(a,(b, \kappa(b)))$ is arbitrarily close to $\eta$. By the definition of $\mathcal{R}^{*}$ and the fact that $\epsilon^{\prime}, \epsilon^{*}$ is arbitrarily close to zero, we get $v^{*}$ respects $\succ$.

Let $u^{*}$ be such that for each $c \in A$,

$$
u_{c}^{*}:=v_{c}^{*}+\kappa(a)-v_{a}^{*}=v_{c}^{*}+\kappa(a)-\eta+\epsilon^{*} .
$$

Since $v^{*}$ respects $\succ$, we have $u^{*}$ respects $\succ$. Further, since $\kappa(a)>\eta$ (Inequality 1), we get that $u^{*}>v^{*}$. Finally, for any $x, y \in A$, we have

$$
\left[v_{x}^{*}-v_{y}^{*}\right]-\left[u_{x}^{*}-u_{y}^{*}\right]=0 .
$$

Thus, $v^{*}$ and $u^{*}$ are $\epsilon$-parallel and pairwise consistent. By $\mathrm{TP}^{\epsilon}$ richness, there is a preference $R^{*} \in \mathcal{R}^{\succ}$ such that $v^{*}, u^{*} \in \mathcal{I}\left(R^{*}\right)$. Notice that $R^{*} \in \mathcal{R}^{*}$. 
STEP 4. In this step, we construct two more vectors which are $\epsilon$-parallel and pairwise consistent for $\succ$. Let $v^{* *} \in \mathbb{R}^{|A|}$ be a vector defined as follows: for each $c \in A$,

$$
v_{c}^{* *}= \begin{cases}v_{a}^{*}+2 \epsilon^{*}=\eta+\epsilon^{*} & \text { if } c=a \\ v_{c}^{*} & \text { otherwise. }\end{cases}
$$

Let $u^{* *} \in \mathbb{R}^{|A|}$ be a vector defined as follows: for each $c \in A$,

$$
u_{c}^{* *}= \begin{cases}u_{a}^{*}=\kappa(a) & \text { if } c=a \\ u_{c}^{*}+\epsilon^{*} & \text { otherwise. }\end{cases}
$$

Since $v^{*}$ and $u^{*}$ respect $\succ$ and pairwise consistent, and $\epsilon^{*}$ is arbitrarily close to zero, we get that $v^{* *}$ and $u^{* *}$ respect $\succ$ and pairwise consistent. Further, for any $x, y \in A \backslash\{a\}$, we have

$$
\left[v_{x}^{* *}-v_{y}^{* *}\right]-\left[u_{x}^{* *}-u_{y}^{* *}\right]=\left[v_{x}^{*}-v_{y}^{*}\right]-\left[u_{x}^{*}-u_{y}^{*}\right]=0 .
$$

For each $x \in A \backslash\{a\}$, we have

$$
\left[v_{a}^{* *}-v_{x}^{* *}\right]-\left[u_{a}^{* *}-u_{x}^{* *}\right]=\left[v_{a}^{*}-v_{x}^{*}+2 \epsilon^{*}\right]-\left[u_{a}^{*}-\left(u_{x}^{*}+\epsilon^{*}\right)\right]=3 \epsilon^{*}<\epsilon,
$$

where the strict inequality followed from the choice of $\epsilon^{*} \in\left(0, \frac{\epsilon}{3}\right)$. Therefore, $v^{* *}$ and $u^{* *}$ are $\epsilon$-parallel. By $\mathrm{TP}^{\epsilon}$ richness, there is a preference $R^{* *} \in \mathcal{R}^{\succ}$ such that $v^{* *}, u^{* *} \in \mathcal{I}\left(R^{* *}\right)$. Notice that $R^{* *} \in \mathcal{R}^{*}$.

The two preferences $R^{*}$ and $R^{* *}$ are illustrated in Figure 8 .

STEP 5. In this step, we show that $f\left(R^{*}\right)=b$ and $f\left(R^{* *}\right)=a$. By definition, $R^{*}, R^{* *} \in \mathcal{R}^{*}$, and by Step 2, $f\left(R^{*}\right), f\left(R^{* *}\right) \in\{a, b\}$. Note that $V^{R^{*}}(a,(b, \kappa(b)))=v_{a}^{*}=\eta-\epsilon^{*}<\eta$. By the definition of $\eta$, there is $\hat{R} \in \mathcal{R}^{*}$ with $f(\hat{R})=b$ such that $V^{\hat{R}}(a,(b, \kappa(b)))>V^{R^{*}}(a,(b, \kappa(b)))$. By monotonicity, $f\left(R^{*}\right) \neq a$. Hence, $f\left(R^{*}\right)=b$.

Next, $V^{R^{* *}}(a,(b, \kappa(b)))=v_{a}^{* *}=\eta+\epsilon^{*}>\eta$. By definition of $\eta, f\left(R^{* *}\right) \neq b$. Hence, $f\left(R^{* *}\right)=a$.

This leads us to a contradiction as follows:

$$
V^{R^{* *}}(b,(a, \kappa(a)))=u_{b}^{* *}=u_{b}^{*}+\epsilon>u_{b}^{*}=V^{R^{*}}(b,(a, \kappa(a))) .
$$

By monotonicity, $f\left(R^{*}\right)=b$ and $f\left(R^{* *}\right)=a$ gives us a contradiction.

Proof of Theorem 2 


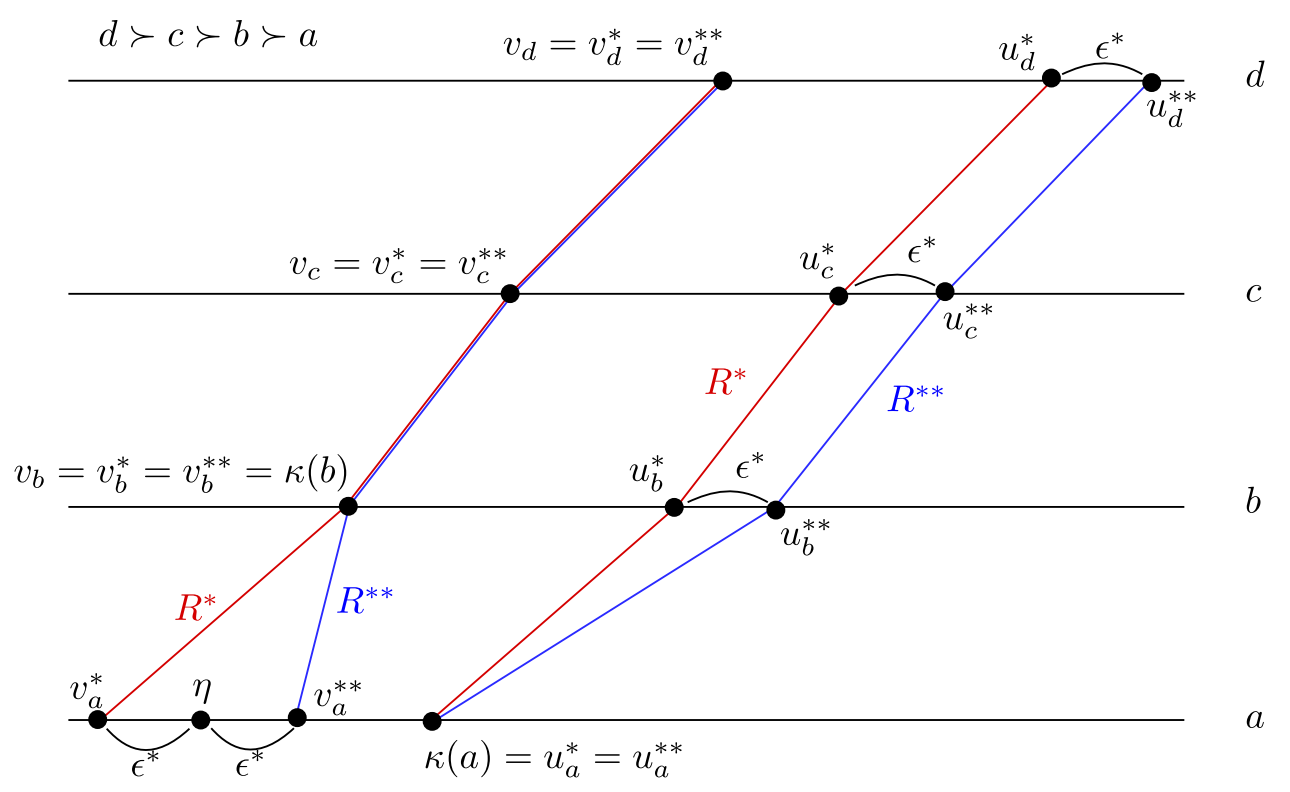

Figure 8: Illustration of $R^{*}$ and $R^{* *}$

Proof: $\quad$ Suppose $(f, p)$ and $\left(f, p^{\prime}\right)$ are two incentive compatible mechanisms. Theorem 1 implies that there are two maps $\kappa: A \rightarrow \mathbb{R}$ and $\kappa^{\prime}: A \rightarrow \mathbb{R}$ such that for all $R, p(R)=$ $\kappa(f(R))$ and $p^{\prime}(R)=\kappa^{\prime}(f(R))$.

Pick $a, b \in A$ such that $b \succ a$. Since $(f, p)$ is incentive compatible, for any $R$ with $f(R)=a$, we have

$$
\left(b, V^{R}(b,(a, \kappa(a)))\right) I(a, \kappa(a)) R(b, \kappa(b)) .
$$

This implies that $V^{R}(b,(a, \kappa(a))) \leq \kappa(b)$. But $b \succ a$ implies that $V^{R}(b,(a, \kappa(a)))>\kappa(a)$. Hence, $\kappa(b)>\kappa(a)$.

A consequence of this observation is that the vector $v$, defined as $v_{c}=\kappa(c)$ for all $c \in A$, respects $\succ$. Similarly, the vector $v^{\prime}$, defined as $v_{c}^{\prime}=\kappa^{\prime}(c)$ for all $c \in A$ respects $\succ$.

Now, if $\kappa(a)=\kappa^{\prime}(a)$ for every $a \in A$, then we are done. So suppose $\kappa(a) \neq \kappa^{\prime}(a)$ for some $a \in A$. Without loss of generality, assume that $\kappa(a)>\kappa^{\prime}(a)$. First we show that $\kappa(b)>\kappa^{\prime}(b)$ for all $b \in A$. Assume for contradiction, $\kappa(b) \leq \kappa^{\prime}(b)$ for some $b \in A$. Let $v \in \mathbb{R}^{|A|}$ be such that $v_{a} \in\left(\kappa^{\prime}(a), \kappa(a)\right), v_{b}=\kappa(b)$, and $v_{c}<\kappa(c)$ for all $c \neq a, b$. Notice that we can take such a $v$ so that it respects $\succ$. By TP ${ }^{\epsilon}$ richness, there is $R \in \mathcal{R}^{\succ}$ such that $v \in \mathcal{I}(R)$.

For each $c \neq b$,

$$
(b, \kappa(b)) P(c, \kappa(c)) .
$$

Thus, incentive compatibility of $(f, p)$ implies $f(R)=b$. However, since

$$
\left(a, \kappa^{\prime}(a)\right) P(b, \kappa(b)) R\left(b, \kappa^{\prime}(b)\right)
$$


incentive compatibility of $\left(f, p^{\prime}\right)$ implies $f(R) \neq b$. This is a contradiction. Hence, $\kappa(b)>$ $\kappa^{\prime}(b)$ for all $b \in A$.

Define $\delta=\min _{a \in A}\left[\kappa(a)-\kappa^{\prime}(a)\right]$ and let $\delta=\kappa\left(a^{*}\right)-\kappa^{\prime}\left(a^{*}\right)$ for some $a^{*} \in A$. Choose $\epsilon^{\prime}>0$ but arbitrarily close to zero. We define two vectors now:

$$
\begin{gathered}
v^{\prime}\left(a^{*}\right)=\kappa^{\prime}\left(a^{*}\right), \quad v^{\prime}(a)=\kappa^{\prime}(a)+\frac{\epsilon^{\prime}}{2} \forall a \neq a^{*} . \\
v\left(a^{*}\right)=\kappa^{\prime}\left(a^{*}\right)+\delta=\kappa\left(a^{*}\right), \quad v(a)=\kappa^{\prime}(a)+\delta-\frac{\epsilon^{\prime}}{2} \forall a \neq a^{*} .
\end{gathered}
$$

Note that $v^{\prime}$ and $v$ respect $\succ$ since $\kappa^{\prime}$ respects $\succ$ and $\epsilon^{\prime}$ is arbitrarily close to zero. Further, since $\delta>0$ and $\epsilon^{\prime}$ is arbitrarily close to zero $v>v^{\prime}$. Finally, for any $a, b \in A$,

$$
\left|[v(a)-v(b)]-\left[v^{\prime}(a)-v^{\prime}(b)\right]\right| \leq \epsilon^{\prime} .
$$

Since $\epsilon^{\prime}$ was chosen arbitrarily close to zero, $v$ and $v^{\prime}$ are $\epsilon$-parallel. Then, $\mathrm{TP}^{\epsilon}$ richness guarantees that there is a preference $R \in \mathcal{R}^{\succ}$ such that $v, v^{\prime} \in \mathcal{I}(R)$.

But incentive compatibility of $\left(f, p^{\prime}\right)$ implies $f(R) \neq a^{*}$ - this follows since $v^{\prime} \in \mathcal{I}(R)$ and $v^{\prime}\left(a^{*}\right)=\kappa^{\prime}\left(a^{*}\right), v^{\prime}(a)=\kappa^{\prime}(a)+\frac{\epsilon^{\prime}}{2}$ for all $a \neq a^{*}$. On the other hand, incentive compatibility of $(f, p)$ implies $f(R)=a^{*}$. This follows from the fact $v \in \mathcal{I}(R)$ and

$$
v\left(a^{*}\right)=\kappa^{\prime}\left(a^{*}\right)+\delta=\kappa\left(a^{*}\right), v(a)=\kappa^{\prime}(a)+\delta-\frac{\epsilon^{\prime}}{2}<\kappa(a) \text { for all } a \neq a^{*} .
$$

This is a contradiction.

\section{Proof of Theorem 3}

Proof: Suppose $(f, p)$ is incentive compatible. The fact that $(f, p)$ is WP-monotone and there exists a map $\kappa: A \rightarrow \mathbb{R}$ such that $p(R)=\kappa(f(R))$ for all $R \in \mathcal{R}$ follow from identical arguments as in Theorem 1 - note here that monotonicity in Theorem 1 implies WP-monotonicity. ${ }^{10}$

We show the payment formula. By incentive compatibility for any $R \in \mathcal{R}$ with $f(R)=1$, we have

$$
(1, \kappa(1)) R(0, \kappa(0)) I\left(1, V^{R}(1,(0, \kappa(0)))\right)
$$

which implies that

$$
\kappa(1) \leq \inf _{R \in \mathcal{R}: f(R)=1} V^{R}(1,(0, \kappa(0))) .
$$

\footnotetext{
${ }^{10}$ Note that the $\mathrm{TP}^{\epsilon}$ richness requirement in Theorem 1 is not required to show the necessity of monotonicity and the taxation principle.
} 
Let

$$
\ell:=\inf _{R \in \mathcal{R}: f(R)=1} V^{R}(1,(0, \kappa(0))) .
$$

We show that $\kappa(1)=\ell$.

Assume for contradiction $\kappa(1)<\ell$. By ontoness, there exists a preference $R$ such that $f(R)=1$. Note that $V^{R}(1,(0, \kappa(0))) \geq \ell$. By ontoness, there is a preference $\hat{R}$ such that $f(\hat{R})=0$. Incentive compatibility of $(f, p)$ implies that $V^{\hat{R}}(1,(0, \kappa(0))) \leq \kappa(1)$.

Now, since $V^{\hat{R}}(1,(0, \kappa(0))) \leq \kappa(1)<\ell \leq V^{R}(1,(0, \kappa(0)))$, by WP-convexity, there is a $\tilde{R}$ such that $V^{\tilde{R}}(1,(0, \kappa(0))) \in(\kappa(1), \ell)$. Since $V^{\tilde{R}}(1,(0, \kappa(0)))>\kappa(1)$, incentive compatibility of $(f, p)$ implies that $f(\tilde{R})=1$. But $V^{\tilde{R}}(1,(0, \kappa(0)))<\ell$ and $f(\tilde{R})=1$ contradicts the definition of $\ell$.

This shows that $\kappa(1)=\ell$. But note that

$$
\begin{aligned}
\ell & =\inf _{R \in \mathcal{R}: f(R)=1} V^{R}(1,(0, \kappa(0))) \\
& =\inf _{R \in \mathcal{R}: f(R)=1}[W P(R, \kappa(0))+\kappa(0)] \\
& =\kappa(0)+\inf _{R \in \mathcal{R}: f(R)=1} W P(R, \kappa(0)) .
\end{aligned}
$$

Then, setting $\tau^{0} \equiv \kappa(0)$ and $\tau^{1} \equiv \inf _{R \in \mathcal{R}: f(R)=1} W P\left(R, \tau^{0}\right)$, gives us the desired payment formula property.

Now, for the converse, suppose $(f, p)$ is WP-monotone and satisfies Property 2(b) of the claim. Pick $R$ with $f(R)=1$ and assume for contradiction $\left(0, \tau^{0}\right) P\left(1, \tau^{0}+\tau^{1}\right)$. Then, $\tau^{0}+\tau^{1}>V^{R}\left(1,\left(0, \tau^{0}\right)\right)=W P\left(R, \tau^{0}\right)+\tau^{0}$, which implies $\tau^{1}>W P\left(R, \tau^{0}\right)$. This violates the definition of $\tau^{1}$.

Next, pick $R$ with $f(R)=0$ and assume for contradiction $\left(1, \tau^{1}+\tau^{0}\right) P\left(0, \tau^{0}\right)$. Then, $\tau^{0}>V^{R}\left(0,\left(1, \tau^{0}+\tau^{1}\right)\right)$. As a result, $V^{R}\left(1,\left(0, \tau^{0}\right)\right)>\tau^{0}+\tau^{1}$ or $W P\left(R, \tau^{0}\right)>\tau^{1}$. By definition of $\tau^{1}$, there is $R^{\prime}$ with $f\left(R^{\prime}\right)=1$ and $W P\left(R^{\prime}, \tau^{0}\right)$ arbitrarily close to $\tau^{1}$. In particular, we can choose $R^{\prime}$ such that $W P\left(R^{\prime}, \tau^{0}\right)<W P\left(R, \tau^{0}\right)$ (this can be done because $W P\left(R, \tau^{0}\right)>\tau^{1}$ and WP-convexity). But $f\left(R^{\prime}\right)=1$ and $f(R)=0$ while $W P\left(R^{\prime}, \tau^{0}\right)<$ $W P\left(R, \tau^{0}\right)$ violates WP-monotonicity, giving us the desired contradiction.

\section{Proof of Theorem 4}

Proof: Let $f$ be an onto allocation rule on $\mathcal{R}$, where $\mathcal{R}^{++} \subseteq \mathcal{R}$. Note that since $\mathcal{R}^{++} \subseteq \mathcal{R}$, the domain $\mathcal{R}$ satisfies WP-convexity - this follows from the fact that for every $t$ and $\alpha \in \mathbb{R}_{++}$, there exists a positive income effect preference $R$ with $W P(R, t)=\alpha$. Hence, Theorem 3 holds in domain $\mathbb{R}$. 
If $(f, p)$ and $\left(f, p^{\prime}\right)$ are incentive compatible mechanisms, then by Theorem 3 , there exists real numbers $\tau^{0}$ and $\bar{\tau}^{0}$ such that for all $R \in \mathcal{R}, p(R)=\tau^{0}$ and $p^{\prime}(R)=\bar{\tau}^{0}$ if $f(R)=0$. We show that $\tau^{0}=\bar{\tau}^{0}$, and by Theorem 3, we will be done.

Assume without loss of generality $\tau^{0}<\bar{\tau}^{0}$. For every $R \in \mathcal{R}$, let $p(R)=\tau^{0}+\tau^{1}$ and $p^{\prime}(R)=\bar{\tau}(0)+\bar{\tau}(1)$ if $f(R)=1$, where $\tau^{1}$ and $\bar{\tau}^{1}$ are as defined in Theorem 3. We consider two possible cases.

CASE 1. Suppose $\bar{\tau}^{1} \geq \tau^{1}$. Construct a positive income effect classical type $R$ such that

$$
W P\left(R, \bar{\tau}^{0}\right)=\bar{\tau}^{1}-\epsilon, \quad W P\left(R, \tau^{0}\right)=\bar{\tau}^{1}+\epsilon,
$$

for some arbitrarily small $\epsilon>0$. Notice that $W P\left(R, \bar{\tau}^{0}\right)<W P\left(R, \tau^{0}\right)$. Since $\mathcal{R}$ consists of all positive income effect classical types, such a $R$ exists. Since $W P\left(R, \bar{\tau}^{0}\right)<\bar{\tau}^{1}$, Theorem 3 implies that $f(R)=0$. But $W P\left(R, \tau^{0}\right)>\bar{\tau}^{1} \geq \tau^{1}$ implies $f(R)=1$. This is a contradiction.

Case 2. Suppose $\bar{\tau}^{1}<\tau^{1}$. Construct a positive income effect classical type $R$ such that

$$
W P\left(R, \bar{\tau}^{0}\right)=\bar{\tau}^{1}+\epsilon, \quad W P\left(R, \tau^{0}\right)=\tau^{1}-\epsilon,
$$

for some arbitrarily small $\epsilon>0$. Notice that since $\epsilon>0$ can be chosen arbitrarily small, $W P\left(R, \tau^{0}\right)>W P\left(R, \bar{\tau}^{0}\right)$, and since the type space consist of all positive income effect types, such a $R$ exists.

Since $W P\left(R, \bar{\tau}^{0}\right)>\bar{\tau}^{1}$, Theorem 3 implies that $f(R)=1$. On the other hand, since $W P\left(R, \tau^{0}\right)<\tau^{1}$, we have $f(R)=0$. This is a contradiction.

Contradictions in all possible cases establish that $\tau^{0}=\bar{\tau}^{0}$. Hence, $p=p^{\prime}$.

\section{Proof of Theorem 5}

Proof: Suppose $(f, p)$ is an incentive compatible mechanism. Note that by Theorem 1, there exists a map $\kappa: A \rightarrow \mathbb{R}$ such that $p(R)=\kappa(f(R))$ for all $R$. ${ }^{11}$ By Lemma 1 , we know that $\kappa\left(a_{0}\right) \leq 0$. If $\kappa\left(a_{0}\right)=0$, then we are done. Hence, assume that $\kappa\left(a_{0}\right)<0$.

We now define another mechanism by defining another map $\tilde{\kappa}: A \rightarrow \mathbb{R}$ as follows. We first set $\tilde{\kappa}\left(a_{0}\right)=0$. Now, we define the rest of the values of $\tilde{\kappa}$ inductively. Denote $A \backslash\left\{a_{0}\right\} \equiv$ $\left\{a_{1}, \ldots, a_{K}\right\}$ and without loss of generality assume that $\kappa\left(a_{1}\right) \leq \kappa\left(a_{2}\right) \leq \ldots \leq \kappa\left(a_{K}\right)$.

\footnotetext{
${ }^{11}$ This is a necessary condition for incentive compatibility and does not requires the $\mathrm{TP}^{\epsilon}$ richness of Theorem 1.
} 
Now, we have defined $\tilde{\kappa}\left(a_{0}\right)=0$. Define $\tilde{\kappa}\left(a_{1}\right)$ as:

$$
\tilde{\kappa}\left(a_{1}\right):=\inf \left\{V^{R}\left(a_{1},\left(a_{0}, \tilde{\kappa}\left(a_{0}\right)\right)\right): f(R)=a_{1}\right\}
$$

Having defined $\tilde{\kappa}\left(a_{1}\right), \ldots, \tilde{\kappa}\left(a_{k}\right)$, we define $\tilde{\kappa}\left(a_{k+1}\right)$ as:

$$
\tilde{\kappa}\left(a_{k+1}\right):=\inf \left\{V^{R}\left(a_{k+1},\left(a_{j}, \tilde{\kappa}\left(a_{j}\right)\right)\right): f(R)=a_{k+1} \text { and } a_{j} \in\left\{a_{0}, a_{1}, \ldots, a_{k}\right\}\right\} .
$$

The mechanism $(\tilde{f}, \tilde{p})$ is defined using $\tilde{\kappa}$ as follows. For every $R$, the agent chooses one of the best bundles in

$$
\left\{\left(a_{0}, 0\right),\left(a_{1}, \tilde{\kappa}\left(a_{1}\right)\right), \ldots,\left(a_{K}, \tilde{\kappa}\left(a_{K}\right)\right)\right\}
$$

according to $R$, where ties are broken in favor of higher indexed alternative. More formally,

$$
M(R):=\left\{\left(a_{j}, \tilde{\kappa}\left(a_{j}\right)\right):\left(a_{j}, \tilde{\kappa}\left(a_{j}\right)\right) R\left(a_{k}, \tilde{\kappa}\left(a_{k}\right)\right) \forall a_{k} \in A\right\}
$$

Then, $\tilde{f}(R)=a_{\ell}$ if $a_{\ell} \in M(R)$ and $\ell \geq j$ for all $a_{j} \in M(R)$.

Suppose the chosen best bundle at $R$ is $\left(a_{k}, \tilde{\kappa}\left(a_{k}\right)\right)$, then $\tilde{f}(R)=a_{k}$ and $\tilde{p}(R)=\tilde{\kappa}\left(a_{k}\right)$. Trivially, this mechanism is incentive compatible since $(\tilde{f}(R), \tilde{p}(R)) \in M(R)$ for all $R$. Further, since $\tilde{\kappa}\left(a_{0}\right)=0$, Lemma 1 implies that it is individually rational. We now complete the proof by showing $\tilde{p}(R) \geq p(R)$ for all $R$.

STEP 1. In this step, we show that $\tilde{\kappa}(a) \geq \kappa(a)$ for all $a \in A$. By definition $\tilde{\kappa}\left(a_{0}\right)>\kappa\left(a_{0}\right)$. Also, by incentive compatibility of $(f, p)$, for every $R$ with $f(R)=a_{1}$, we have $\kappa\left(a_{1}\right) \leq$ $V^{R}\left(a_{1},\left(a_{0}, \kappa\left(a_{0}\right)\right)\right)$. Since $\tilde{\kappa}\left(a_{0}\right)=0>\kappa\left(a_{0}\right)$, we get $\kappa\left(a_{1}\right)<V^{R}\left(a_{1},\left(a_{0}, 0\right)\right)$. Hence, we get

$$
\kappa\left(a_{1}\right) \leq \inf _{R: f(R)=a_{1}} V^{R}\left(a_{1},\left(a_{0}, 0\right)\right)=\tilde{\kappa}\left(a_{1}\right) .
$$

Now, assume that $\kappa\left(a_{j}\right) \leq \tilde{\kappa}\left(a_{j}\right)$ for all $j \in\{1, \ldots, k\}$. We establish that $\kappa\left(a_{k+1}\right) \leq$ $\tilde{\kappa}\left(a_{k+1}\right)$. Pick any $R$ such that $f(R)=a_{k+1}$. For every $a_{j} \in\left\{a_{0}, a_{1}, \ldots, a_{k}\right\}$, incentive compatibility of $(f, p)$ implies that

$$
\kappa\left(a_{k+1}\right) \leq V^{R}\left(a_{k+1},\left(a_{j}, \kappa\left(a_{j}\right)\right)\right) .
$$

As a result, we get

$$
\kappa\left(a_{k+1}\right) \leq \inf \left\{V^{R}\left(a_{k+1},\left(a_{j}, \kappa\left(a_{j}\right)\right)\right): f(R)=a_{k+1}, a_{j} \in\left\{a_{0}, a_{1}, \ldots, a_{k}\right\} .\right.
$$

By induction, $\kappa\left(a_{j}\right) \leq \tilde{\kappa}\left(a_{j}\right)$ for all $a_{j} \in\left\{a_{0}, a_{1}, \ldots, a_{k}\right\}$. This implies that for every $R$ with $f(R)=a_{k+1}$ and for every $a_{j} \in\left\{a_{0}, a_{1}, \ldots, a_{k}\right\}$,

$$
V^{R}\left(a_{k+1},\left(a_{j}, \kappa\left(a_{j}\right)\right)\right) \leq V^{R}\left(a_{k+1},\left(a_{j}, \tilde{\kappa}\left(a_{j}\right)\right)\right)
$$


But then,

$$
\kappa\left(a_{k+1}\right) \leq \inf \left\{V^{R}\left(a_{k+1},\left(a_{j}, \tilde{\kappa}\left(a_{j}\right)\right)\right): f(R)=a_{k+1}, a_{j} \in\left\{a_{0}, a_{1}, \ldots, a_{k}\right\}\right\}=\tilde{\kappa}\left(a_{k+1}\right) .
$$

This completes the proof of this step.

STEP 2. We complete the proof in this step. Consider $R$ and denote $f(R)=a_{k}$. By definition, $\tilde{\kappa}\left(a_{k}\right) \leq V^{R}\left(a_{k},\left(a_{j}, \tilde{\kappa}\left(a_{j}\right)\right)\right)$ for all $j<k$. Hence, $\left(a_{k}, \tilde{\kappa}\left(a_{k}\right)\right) R\left(a_{j}, \tilde{\kappa}\left(a_{j}\right)\right)$ for all $j<k$. By our tie-breaking rule for defining $\tilde{f}$, we have $\tilde{f}(R) \neq a_{j}$ for all $j<k$. Hence, $\tilde{f}(R)=a_{\ell}$, where $\ell \geq k$. But

$$
\tilde{p}(R)=\tilde{\kappa}\left(a_{\ell}\right) \geq \kappa\left(a_{\ell}\right) \geq \kappa\left(a_{k}\right)=p(R) .
$$

This completes the proof.

Proof of Lemma 2

Proof: Suppose $(f, p)$ is incentive compatible. By Theorem 1 , there exists $\kappa: A \rightarrow \mathbb{R}$ such that $p(R)=\kappa(f(R))$ for all $R$. Just as in the proof of Theorem 2, we can show that for any $a, b \in A$ with $b \succ a$, we have $\kappa(b)>\kappa(a)$.

A consequence of this observation is that the vector $v$, defined as $v_{c}=\kappa(c)$ for all $c \in A$, respects $\succ$. Now, pick any $a \in A$. Choose any $\epsilon>0$ but arbitrarily close to zero. Then, the vector $v^{\prime}$ defined as $v_{c}^{\prime}=v_{c}$ for all $c \neq a$ and $v_{a}^{\prime}=v_{a}+\epsilon$, also respects $\succ$. Hence, by $\mathrm{TP}^{\epsilon}$ richness, there is a preference $R$ such that $v^{\prime} \in \mathcal{I}(R)$. Note that for any $b \neq a$, we have $(a, \kappa(a)) P(b, \kappa(b))$. Hence, incentive compatibility implies that $f(R)=a$. By definition of $v$ and $v^{\prime}$, we get that for all $b \neq a$,

$$
V^{R}(a,(b, \kappa(b)))=\kappa(a)+\epsilon .
$$

Since $\epsilon$ was arbitrarily close to zero, by letting $\epsilon \rightarrow 0$, we get

$$
\kappa(a)=\inf _{R: f(R)=a} V^{R}(a,(b, \kappa(b))) \text { for all } b \in A .
$$

Similarly, we can define $v^{\prime \prime}$ as $v_{c}^{\prime \prime}=v_{c}-\epsilon$ for all $c \neq a$ and $v_{a}^{\prime \prime}=v_{a}$. Note that for sufficiently small $\epsilon>0, v^{\prime \prime}$ respects $\succ$. Hence, by $\mathrm{TP}^{\epsilon}$ richness, there is a preference $R^{\prime \prime}$ such that $v^{\prime \prime} \in \mathcal{I}\left(R^{\prime \prime}\right)$. Note that for any $b \neq a$, we have $(a, \kappa(a)) P^{\prime \prime}(b, \kappa(b))$. Hence, incentive compatibility implies that $f\left(R^{\prime \prime}\right)=a$. By definition of $v$ and $v^{\prime \prime}$, we get that for all $b \neq a$,

$$
V^{R^{\prime \prime}}(b,(a, \kappa(a)))=\kappa(b)-\epsilon
$$


Since $\epsilon>0$ was arbitrarily close to zero, by letting $\epsilon \rightarrow 0$, we get

$$
\kappa(b)=\sup _{R^{\prime \prime}: f\left(R^{\prime \prime}\right)=a} V^{R^{\prime \prime}}(b,(a, \kappa(a))) .
$$

For the converse, choose any $R, R^{\prime} \in \mathcal{R}^{\succ}$ and we get,

$$
p(R) \leq V^{R}\left(f(R),\left(f\left(R^{\prime}\right), p\left(R^{\prime}\right)\right)\right) .
$$

Hence, $(f(R), p(R)) R\left(f\left(R^{\prime}\right), p\left(R^{\prime}\right)\right)$. This shows that $(f, p)$ is incentive compatible.

\section{Proofs of Section 6}

\section{Proof of Proposition 1}

Proof: We show that $\mathcal{R}_{r}^{\succ, \epsilon}$ satisfies $\mathrm{TP}^{\epsilon}$-richness, and the result follows from Theorems 1 and 2. Pick $v, v^{\prime} \in \mathbb{R}^{|A|}$ such that $v, v^{\prime}$ are $\epsilon$-parallel and pairwise consistent for $\succ$. Assume without loss of generality that $v<v^{\prime}$. We first construct a preference $R \in \mathcal{R}_{r}^{\succ}$. This is done by specifying $\nu$ and $B$. We let $B$ to be a large positive number such that $B>\max _{a \in A} v^{\prime}(a)$. We let $\nu\left(a_{0}\right)=0$ and $\nu(b)=v(b)-v\left(a_{0}\right)$ for all $b \neq a_{0}$. So, $R \in \mathcal{R}_{r}^{\succ}$ is a preference generated from this choice of $(\nu, B)$. Similarly, define $R^{\prime}$ as follows: $B$ is same as before but $\nu^{\prime}\left(a_{0}\right)=0$ and $\nu^{\prime}(b)=v^{\prime}(b)-v^{\prime}\left(a_{0}\right)$ for all $b \neq a_{0}$. Hence, $\left(\nu^{\prime}, B\right)$ defines $R^{\prime} \in \mathcal{R}_{r}^{\succ}$. Notice that since $v$ and $v^{\prime}$ are $\epsilon$-parallel, $d\left(R, R^{\prime}\right)<\epsilon$.

Now, we construct another preference $\bar{R}$ as follows. This is done by specifying the indifference vectors of $\bar{R}$. For all $v^{\prime \prime}<v$, we have $v^{\prime \prime} \in \mathcal{I}(\bar{R})$ if and only if $v^{\prime \prime} \in \mathcal{I}(R)$. For all $v^{\prime \prime}>v^{\prime}$, we have $v^{\prime \prime} \in \mathcal{I}(\bar{R})$ if and only if $v^{\prime \prime} \in \mathcal{I}\left(R^{\prime}\right)$. For all $v \leq v^{\prime \prime} \leq v^{\prime}$, we have $v^{\prime \prime} \in \mathcal{I}(\bar{R})$ if and only if there exists $\alpha \in[0,1]$ such that $v^{\prime \prime}=\alpha v+(1-\alpha) v^{\prime}$. Informally, indifference vectors to the left of $v$ coincide with the indifference vector of $R$, to the right of $v^{\prime}$ coincide with the indifference vector of $R^{\prime}$, and it is a "convex combination" of indifference vectors of $R$ and $R^{\prime}$ between $v$ and $v^{\prime}$. It is clear that $\bar{R}$ exists and belongs to $\mathcal{R}^{\succ}$.

The fact that $d(\bar{R}, R)<\epsilon$ follows from the fact that $v$ and $v^{\prime}$ are $\epsilon$-parallel and $d\left(R, R^{\prime}\right)<$ $\epsilon$. We formalize this intuition below. Choose any $a \neq a_{0}$.

For $t<v\left(a_{0}\right), V^{R}\left(a,\left(a_{0}, t\right)\right)-V^{\bar{R}}\left(a,\left(a_{0}, t\right)\right)=0$. For $t>v^{\prime}\left(a_{0}\right), V^{R^{\prime}}\left(a,\left(a_{0}, t\right)\right)-$ $V^{\bar{R}}\left(a,\left(a_{0}, t\right)\right)=0$. Now, for the final case, consider $t \in\left[v\left(a_{0}\right), v^{\prime}\left(a_{0}\right)\right]$. Suppose $\alpha \in[0,1]$ is 
such that $t=\alpha v\left(a_{0}\right)+(1-\alpha) v^{\prime}\left(a_{0}\right)$. Note that $B>t$. Hence,

$$
\begin{aligned}
& V^{R}\left(a,\left(a_{0}, t\right)\right)-V^{\bar{R}}\left(a,\left(a_{0}, t\right)\right) \\
& =\left[v(a)+t-v\left(a_{0}\right)\right]-\left[\alpha v(a)+(1-\alpha) v^{\prime}(a)\right] \\
& =\left[v(a)-v\left(a_{0}\right)+\alpha v\left(a_{0}\right)+(1-\alpha) v^{\prime}\left(a_{0}\right)\right]-\left[\alpha v(a)+(1-\alpha) v^{\prime}(a)\right] \\
& =(1-\alpha)\left(\left[v(a)-v\left(a_{0}\right)\right]-\left[v^{\prime}(a)-v^{\prime}\left(a_{0}\right)\right]\right) \\
& <\epsilon
\end{aligned}
$$

where the second equality follows from the definition of $t$ and the inequality follows from the fact that $v$ and $v^{\prime}$ are $\epsilon$-parallel and $\alpha \in[0,1]$. Hence, $d(R, \bar{R})<\epsilon$.

\section{Proof of Theorem 6}

Proof: One direction is easy and skipped. For the other direction, we propose a straightforward extension of Theorem 3 to the $n$-agent case. For completeness, we state the result without a proof.

TheOREM 7 ( $\boldsymbol{n}$-agent Theorem 3) Suppose $\mathcal{R}$ is a classical type space satisfying strong WP-convexity and $(f, \mathbf{p})$ is a mechanism defined on $\mathcal{R}^{n}$. Then, $(f, \mathbf{p})$ is DSIC if and only if for every agent $i \in N$, there exists a map $\tau_{i}^{0}: \mathcal{R}^{n-1} \rightarrow \mathbb{R}$ such that for every $R_{-i} \in \mathcal{R}^{n-1}$,

- WP-Monotonicity. for every $R_{i}, R_{i}^{\prime} \in \mathcal{R}$ with $f_{i}\left(R_{i}, R_{-i}\right)=1$,

$$
\left[W P\left(R_{i}^{\prime}, \tau_{i}^{0}\left(R_{-i}\right)\right)>W P\left(R_{i}, \tau_{i}^{0}\left(R_{-i}\right)\right)\right] \Rightarrow f_{i}\left(R_{i}^{\prime}, R_{-i}\right)=1
$$

- Payment formula. for every $R_{i} \in \mathcal{R}$,

$$
p_{i}\left(R_{i}, R_{-i}\right)=\tau_{i}^{0}\left(R_{-i}\right)+f_{i}\left(R_{i}, R_{-i}\right) \cdot \tau_{i}^{1}\left(R_{-i}\right)
$$

where $\tau_{i}^{1}\left(R_{-i}\right)=\inf \left\{W P\left(R_{i}^{\prime}, \tau_{i}^{0}\left(R_{-i}\right)\right): R_{i}^{\prime} \in \mathcal{R}, f_{i}\left(R_{i}^{\prime}, R_{-i}\right)=1\right\}$

Though Theorem 7 is a simple extension of Theorem 3 to the multi-agent case, it shows the informational complexity of an incentive compatible mechanism in the multi-agent model. Now, $\tau_{i}^{0}$ and $\tau_{i}^{1}$ are maps that depend on the preferences of other agents. As a result, the mechanism must elicit infinite number of willingness to pay of each agent. In contrast, a one-agent incentive compatible mechanism only elicits one value of willingness to pay.

Now, let $(f, \mathbf{p})$ be a DSIC, anonymous mechanism on a rich classical type space $\mathcal{R}$ satisfying LPI. By Theorem 7 and LPI property, there exists $\tau^{0}$ such that for every $i \in N$ and 
for all $\mathbf{R} \in \mathcal{R}, f_{i}(\mathbf{R})=0$ implies $p_{i}(\mathbf{R})=\tau^{0}$ and $f_{i}(\mathbf{R})=1$ implies $p_{i}(\mathbf{R})=\tau^{0}+\tau_{i}^{1}\left(R_{-i}\right)$, where $\tau_{i}^{1}\left(R_{-i}\right)$ is as defined in Theorem 7 . We now do the proof in three steps.

SteP 1. Consider the following property.

Definition 20 A mechanism $(f, \mathbf{p})$ satisfying LPI $\left(a t \tau^{0}\right)$ is restricted WP-efficient if for all $\mathbf{R} \in \mathcal{R}$ with $f_{i}(\mathbf{R})=1$ for some $i \in N$, we have $W P\left(R_{i}, \tau^{0}\right) \geq W P\left(R_{j}, \tau^{0}\right)$ for all $j \in N$.

We show that $(f, \mathbf{p})$ is restricted WP-efficient. Assume for contradiction that there is a type profile $\mathbf{R}$ such that $f_{i}(\mathbf{R})=1$ and $W P\left(R_{i}, \tau^{0}\right)<W P\left(R_{j}, \tau^{0}\right)$ for some $j \neq i$. Consider $R_{i}^{\prime}=R_{j}$. By definition $W P\left(R_{i}^{\prime}, \tau^{0}\right)>W P\left(R_{i}, \tau^{0}\right)$ and monotonicity in Theorem 7 implies that $f_{i}\left(R_{i}^{\prime}, R_{j}, R_{-i j}\right)=1$. Further, $W P\left(R_{i}^{\prime}, \tau^{0}\right)>W P\left(R_{i}, \tau^{0}\right) \geq \tau_{i}^{1}\left(R_{-i}\right)$. By anonymity,

$$
\left(1, \tau^{0}+\tau_{i}^{1}\left(R_{-i}\right)\right) I_{j}\left(0, \tau^{0}\right) I_{j}\left(1, \tau^{0}+W P\left(R_{j}, \tau^{0}\right)\right) .
$$

This implies that $\tau_{i}^{1}\left(R_{-i}\right)=W P\left(R_{j}, \tau^{0}\right)=W P\left(R_{i}^{\prime}, \tau^{0}\right)$, a contradiction.

SteP 2. In this step, we define the reserve price map. Consider two agents $i$ and $j$. Fix a type $R_{0} \in \mathcal{R}$ and $R_{-i j} \in \mathcal{R}^{n-1}$. Notationally, whenever we write a type profile in this step, we first write the type of agent $i$, followed by the type of agent $j$, and then $R_{-i j}$. We first show that there is $R_{i} \in \mathcal{R}$ such that $f_{i}\left(R_{i}, R_{0}, R_{-i j}\right)=1$ if and only if there is $R_{j} \in \mathcal{R}$ such that $f_{j}\left(R_{0}, R_{j}, R_{-i j}\right)=1$. To show this, suppose $f_{i}\left(R_{i}, R_{0}, R_{-i j}\right)=1$. By Theorem 7 , we can choose $R_{i}$ such that $W P\left(R_{i}, \tau^{0}\right)>\tau_{i}^{1}\left(R_{0}, R_{-i j}\right)$. Now, consider the type profile $\left(R_{0}, R_{j}, R_{-i j}\right)$, where $R_{j}=R_{i}$. If $f_{j}\left(R_{0}, R_{j}, R_{-i j}\right)=0$, then anonymity implies

$$
\left(1, \tau^{0}+W P\left(R_{j}, \tau^{0}\right)\right) I_{j}\left(0, \tau^{0}\right) I_{j}\left(1, \tau^{0}+\tau^{1}\left(R_{0}, R_{-i j}\right)\right) .
$$

But this implies that $\tau_{i}^{1}\left(R_{0}, R_{-i j}\right)=W P\left(R_{j}, \tau^{0}\right)=W P\left(R_{i}, \tau^{0}\right)$, a contradiction.

Hence, there is $R_{i} \in \mathcal{R}$ such that $f_{i}\left(R_{i}, R_{0}, R_{-i j}\right)=1$ if and only if there is $R_{j} \in \mathcal{R}$ such that $f_{j}\left(R_{0}, R_{j}, R_{-i j}\right)=1$. Now, suppose that there is $R_{i} \in \mathcal{R}$ such that $f_{i}\left(R_{i}, R_{0}, R_{-i j}\right)=1$. We show that $\tau_{i}^{1}\left(R_{0}, R_{-i j}\right)=\tau_{j}^{1}\left(R_{0}, R_{-i j}\right)$. First, note that by strong WP-convexity and Theorem 7, we can assume $W P\left(R_{i}, \tau^{0}\right)>\max \left\{\tau_{i}^{1}\left(R_{0}, R_{-i j}\right), \tau_{j}^{1}\left(R_{0}, R_{-i j}\right)\right\}$ without loss of generality. Consider the type profile $\left(R_{0}, R_{j}^{\prime}, R_{-i j}\right)$, where $R_{j}^{\prime}=R_{i}$. Note that since $W P\left(R_{j}^{\prime}, \tau^{0}\right)=$ $W P\left(R_{i}, \tau^{0}\right)>\tau_{j}^{1}\left(R_{0}, R_{-i j}\right)$, by Theorem $7, f_{j}\left(R_{0}, R_{j}^{\prime}, R_{-i j}\right)=1$. By anonymity,

$$
\left(1, \tau^{0}+\tau_{i}^{1}\left(R_{0}, R_{-i j}\right)\right) I_{i}\left(1, \tau^{0}+\tau_{j}^{1}\left(R_{0}, R_{-i j}\right)\right) .
$$

Hence, $\tau_{i}^{1}\left(R_{0}, R_{-i j}\right)=\tau_{j}^{1}\left(R_{0}, R_{-i j}\right)$. 
Now, if there is $R_{i} \in \mathcal{R}$ such that $f_{i}\left(R_{i}, R_{0}, R_{-i j}\right)=1$, then define $r\left(R_{0}, R_{-i j}\right)=$ $\tau_{i}^{1}\left(R_{0}, R_{-i j}\right)=\tau_{j}^{1}\left(R_{0}, R_{-i j}\right)$. If $f_{i}\left(R_{i}, R_{0}, R_{-i j}\right)=0$ for all $R_{i} \in \mathcal{R}$, then define $r\left(R_{0}, R_{-i j}\right)=$ $\beta_{\tau^{0}}$, where we had assumed that the willingness to pay at transfer level $\tau^{0}$ lies in $\left(0, \beta_{\tau^{0}}\right)$.

STEP 3. We now verify the three conditions in the definition of AVVR mechanism with respect to the reserve price map $r$ defined in Step 2. Fix a type profile $\mathbf{R} \in \mathcal{R}^{n}$.

First, suppose $W(\mathbf{R} ; r)=\emptyset$. Assume for contradiction for some $i \in N, f_{i}(\mathbf{R})=1$. Then, by definition, $r\left(R_{-i}\right)=\tau_{i}^{1}\left(R_{-i}\right)$. Hence, by Theorem $7, W P\left(R_{i}, \tau^{0}\right) \geq \tau_{i}^{1}\left(R_{-i}\right)=r\left(R_{-i}\right)$. Step 1 implies that $W P\left(R_{i}, \tau^{0}\right) \geq W P\left(R_{j}, \tau^{0}\right)$ for all $j \in N$. Hence, $i \in W(\mathbf{R} ; r)$, which is a contradiction.

Second, let $i \in W^{*}(\mathbf{R} ; r)$. Then, by definition of $W^{*}(\mathbf{R} ; r)$, we have $W P\left(R_{i}, \tau^{0}\right)>r\left(R_{-i}\right)$. If $r\left(R_{-i}\right)=\beta_{\tau^{0}}$, then $W P\left(R_{i}, \tau^{0}\right)>\beta_{\tau^{0}}$, which contradicts the fact that $W P$ values at $\tau^{0}$ lie in $\left(0, \beta_{\tau^{0}}\right)$. Hence, $r\left(R_{-i}\right)=\tau_{i}^{1}\left(R_{-i}\right)$. This implies that $W P\left(R_{i}, \tau^{0}\right)>\tau_{i}^{1}\left(R_{-i}\right)$. By Theorem $7, f_{i}(\mathbf{R})=1$.

Finally, if $f_{i}(\mathbf{R})=1$ for some $i \in N$, then Theorem 7 implies $W P\left(R_{i}, \tau^{0}\right) \geq \tau_{i}^{1}\left(R_{-i}\right)=$ $r\left(R_{-i}\right)$. Step 1 then implies that $i \in W(\mathbf{R} ; r)$.

We conclude the proof by showing the payment in this mechanism coincides with the payment formula given in the definition of the AVVR mechanism. By Theorem 7 and LPI, if $f_{i}(\mathbf{R})=0$ then $p_{i}(\mathbf{R})=\tau^{0}$. Further, if $f_{i}(\mathbf{R})=1$ then $p_{i}(\mathbf{R})=\tau^{0}+\tau_{i}^{1}\left(R_{-i}\right)=\tau^{0}+r\left(R_{-i}\right)$. We show that $\max _{j \neq i} W P\left(R_{j}, \tau^{0}\right) \leq r\left(R_{-i}\right)$. To see this, suppose $f_{i}(\mathbf{R})=1$ and $r\left(R_{-i}\right)<$ $\max _{j \neq i} W P\left(R_{j}, \tau^{0}\right)$. Then we can choose $R_{i}^{\prime}$ such that $\tau_{i}^{1}\left(R_{-i}\right)=r\left(R_{-i}\right)<W P\left(R_{i}^{\prime}, \tau^{0}\right)<$ $\max _{j \neq i} W P\left(R_{j}, \tau^{0}\right)$. By Theorem $7, f_{i}\left(R_{i}^{\prime}, R_{-i}\right)=1$. But this contradicts restricted WPefficiency in Step 1. Hence, $\max _{j \neq i} W P\left(R_{j}, \tau^{0}\right) \leq r\left(R_{-i}\right)$, and this shows

$$
p_{i}(\mathbf{R})=\tau^{0}+f_{i}(\mathbf{R}) \cdot \max \left(r\left(R_{-i}\right), \max _{j \neq i} W P\left(R_{j}, \tau^{0}\right)\right) .
$$

\section{Proof of Proposition 3}

Proof: Let $\mathcal{R}^{C}$ be the set of all classical preferences. It is enough to show that every MWEP mechanism is DSIC on the domain $\mathcal{R}^{C}$. Let $(f, \mathbf{p})$ be an MWEP mechanism defined on $\mathcal{R}^{C}$. Since $\mathcal{R}^{C}$ satisfies $\mathrm{TP}^{\epsilon}$ richness for any $\epsilon$, by Theorem 1, it is enough to show that $(f, \mathbf{p})$ satisfies taxation principle and monotonicity.

To establish this, we will need a result from the literature. Fix a type profile $\mathbf{R} \equiv$ $\left(R_{1}, \ldots, R_{n}\right)$ and a price vector $\pi \in \mathbb{R}_{+}^{A}$ with $\pi_{a_{0}}=0$. For any $S \subseteq M$, the demanders of 
$S$ are

$$
U(S, \pi ; \mathbf{R}):=\left\{i \in N: D\left(R_{i}, \pi\right) \cap S \neq \emptyset\right\} .
$$

For any $S \subseteq M$, the exclusive demanders of $S$ are

$$
O(S, \pi ; \mathbf{R}):=\left\{i \in N: D\left(R_{i}, \pi\right) \subseteq S\right\}
$$

Morimoto and Serizawa (2015) show that if $\pi$ is the minimum Walrasian equilibrium price vector at $\mathbf{R}$, then

$$
\begin{array}{ll}
|O(S, \pi ; \mathbf{R})| \leq|S| \quad \forall S \subseteq M \\
|U(S, \pi ; \mathbf{R})|>|S| \quad \forall S \subseteq M \text { such that } \pi_{a}>0 \forall a \in S .
\end{array}
$$

The first condition is clearly necessary - if the number of exclusive demanders of $S$ is more than $|S|$, then they cannot be assigned in any Walrasian equilibrium. The necessity of the second condition is non-trivial - intuitively, if the second condition is not true, we can reduce the price of objects in $S$ and get a new Walrasian equilibrium price, contradicting minimality of $\pi .{ }^{12}$

Consider two type profiles $\left(R_{i}, R_{-i}\right)$ and $\left(R_{i}^{\prime}, R_{-i}\right)$ and denote $\pi \equiv \pi^{\min }\left(R_{i}, R_{-i}\right)$ and $\pi^{\prime} \equiv \pi^{\min }\left(R_{i}^{\prime}, R_{-i}\right)$. Suppose $f_{i}\left(R_{i}, R_{-i}\right)=a, f_{i}\left(R_{i}^{\prime}, R_{-i}\right)=b$. Consider the following sets:

$$
M^{\pi<\pi^{\prime}}:=\left\{x \in M: \pi_{x}<\pi_{x}^{\prime}\right\}, \quad M^{\pi>\pi^{\prime}}:=\left\{x \in M: \pi_{x}>\pi_{x}^{\prime}\right\} .
$$

Note that $\pi_{x}>0$ for each $x \in M^{\pi>\pi^{\prime}}$ and $\pi_{x}^{\prime}>0$ for each $x \in M^{\pi<\pi^{\prime}}$. Hence, $a_{0} \notin$ $\left(M^{\pi<\pi^{\prime}} \cup M^{\pi>\pi^{\prime}}\right)$. We prove the following claim.

Claim 1 The following hold: (1) $a \notin M^{\pi<\pi^{\prime}}$ and (2) $b \notin M^{\pi>\pi^{\prime}}$.

Proof: Suppose $a \in M^{\pi<\pi^{\prime}}$. Since $\pi^{\prime}$ is the MWEP at $\left(R_{i}^{\prime}, R_{-i}\right)$ and $\pi_{x}^{\prime}>0$ for each $x \in M^{\pi<\pi^{\prime}}$,

$$
U\left(M^{\pi<\pi^{\prime}}, \pi^{\prime} ;\left(R_{i}^{\prime}, R_{-i}\right)\right) \geq\left|M^{\pi<\pi^{\prime}}\right|+1
$$

Denote $\hat{N}:=U\left(M^{\pi<\pi^{\prime}}, \pi^{\prime} ;\left(R_{i}^{\prime}, R_{-i}\right)\right) \backslash\{i\}$. Since prices of only objects in $M^{\pi<\pi^{\prime}}$ decrease from $\pi^{\prime}$ to $\pi$ and preferences of agents in $\hat{N}$ do not change from $\left(R_{i}, R_{-i}\right)$ to $\left(R_{i}^{\prime}, R_{-i}\right)$, we have $\hat{N} \subseteq O\left(M^{\pi<\pi^{\prime}}, \pi ;\left(R_{i}, R_{-i}\right)\right)$. But Inequality 2 implies that $|\hat{N}| \geq\left|M^{\pi<\pi^{\prime}}\right|$. This in turn implies that no agent outside $\hat{N}$ can be assigned an object in $M^{\pi<\pi^{\prime}}$ in the MWEP $\pi$ at $\left(R_{i}, R_{-i}\right)$. But $i \notin \hat{N}, a \in M^{\pi<\pi^{\prime}}$, and $f_{i}\left(R_{i}, R_{-i}\right)=a$ gives us a contradiction.

\footnotetext{
${ }^{12}$ Morimoto and Serizawa (2015) show that these two conditions are necessary and sufficient for any price vector to be the minimum Walrasian equilibrium price vector.
} 
An analogous proof can be done for $b \notin M^{\pi>\pi^{\prime}}$.

We now complete the proof in two steps.

TAXATION PRINCIPLE. If $a=b$, then by Claim $1, a \notin\left(M^{\pi<\pi^{\prime}} \cup M^{\pi>\pi^{\prime}}\right)$. This implies that $\pi_{a}=\pi_{a}^{\prime}$, and we are done.

Monotonicity. Suppose $f_{i}\left(R_{i}, R_{-i}\right)=a, f_{i}\left(R_{i}^{\prime}, R_{-i}\right)=b$. Assume for contradiction that $V^{R_{i}^{\prime}}\left(b,\left(a, \pi_{a}\right)\right)<V^{R_{i}}\left(b,\left(a, \pi_{a}\right)\right)$. Since $a \in D\left(R_{i}, \pi\right)$, we must have $\pi_{b} \geq V^{R_{i}}\left(b,\left(a, \pi_{a}\right)\right)>$ $V^{R_{i}^{\prime}}\left(b,\left(a, \pi_{a}\right)\right)$. Suppose $\pi_{a}^{\prime} \leq \pi_{a}$, then $b \in D\left(R_{i}^{\prime}, \pi^{\prime}\right)$ implies that $\pi_{b}^{\prime} \leq V^{R_{i}^{\prime}}\left(b,\left(a, \pi_{a}^{\prime}\right)\right)<$ $V^{R_{i}^{\prime}}\left(b,\left(a, \pi_{a}\right)\right)<\pi_{b}$. Hence, $b \in M^{\pi>\pi^{\prime}}$, a contradiction to Claim 1 .

Similarly, if $\pi_{a}^{\prime}>\pi_{a}$, we have $a \in M^{\pi<\pi^{\prime}}$, a contradiction to Claim 1 .

\section{References}

ADACHI, T. (2014): "Equity and the Vickrey allocation rule on general preference domains," Social Choice and Welfare, 42, 813-830.

Ashlagi, I., M. Braverman, A. Hassidim, And D. Monderer (2010): "Monotonicity and implementability," Econometrica, 78, 1749-1772.

Ashlagi, I. AND S. SERIzAWA (2012): "Characterizing Vickrey allocation rule by anonymity," Social Choice and Welfare, 38, 531-542.

Ausubel, L. M. And P. Milgrom (2002): "Ascending auctions with package bidding," Frontiers of Theoretical Economics, 1, 1-42.

BAIsA, B. (2017): "Auction design without quasilinear preferences," Theoretical Economics, $12,53-78$.

- (2016): "Efficient multi-unit auctions for normal goods," Available at SSRN 2824921.

Bikhchandani, S., S. Chatterji, R. Lavi, A. Mualem, N. Nisan, And A. Sen (2006): "Weak monotonicity characterizes deterministic dominant strategy implementation," Econometrica, 74, 1109-1132.

Borgers, T., D. Krahmer, And R. Strausz (2015): An introduction to the theory of mechanism design, Oxford University Press, USA. 
Carbajal, J. C. And J. Ely (2013): "Mechanism design without revenue equivalence," Journal of Economic Theory, 148, 104-133.

Carbajal, J. C. and R. Müller (2015): "Implementability under monotonic transformations in differences," Journal of Economic Theory, 160, 114-131.

Che, Y.-K. And I. Gale (2000): "The optimal mechanism for selling to a budgetconstrained buyer," Journal of Economic Theory, 92, 198-233.

Chung, K.-S. And W. Olszewski (2007): "A non-differentiable approach to revenue equivalence," Theoretical Economics, 2, 1-19.

Clarke, E. (1971): "Multipart pricing of public goods," Public Choice, 11, 17-33.

Cuff, K., S. Hong, J. A. Schwartz, Q. Wen, and J. Weymark (2012): "Dominant strategy implementation with a convex product space of valuations," Social Choice and Welfare, 39, 567-597.

Demange, G. And D. Gale (1985): "The strategy structure of two-sided matching markets," Econometrica, 53, 873-888.

Dobzinski, S., R. LAVI, AND N. NisAn (2012): "Multi-unit auctions with budget limits," Games and Economic Behavior, 74, 486-503.

Garratt, R. And M. Pycia (2016): "Efficient bilateral trade," Working Paper, UCLA.

Groves, T. (1973): "Incentives in teams," Econometrica, 41, 617-631.

Hashimoto, K. And H. Saitoh (2010): "Domain expansion of the pivotal mechanism," Social Choice and Welfare, 34, 455-470.

Heydenreich, B., R. Muller, M. Uetz, and R. V. Vohra (2009): "Characterization of revenue equivalence," Econometrica, 77, 307-316.

Jehiel, P., B. Moldovanu, and E. Stacchetti (1999): "Multidimensional mechanism design for auctions with externalities," Journal Economic Theory, 85, 258-293.

Kazumura, T., D. Mishra, and S. Serizawa (2017): "Multi-object auction design without quasilinearity: Revenue maximization with no wastage," ISER Discussion paper No. 1001. 
Kazumura, T. And S. Serizawa (2016): "Efficiency and strategy-proofness in object assignment problems with multi-demand preferences," Social Choice and Welfare, 47, 633663.

Kos, N. And M. Messner (2013): "Incentive compatibility in non-quasilinear environments," Economics Letters, 121, 12-14.

Krishna, V. And E. MaEnner (2001): "Convex potentials with an application to mechanism design," Econometrica, 69, 1113-1119.

LAVI, R. AND M. MAY (2012): "A note on the incompatibility of strategy-proofness and pareto-optimality in quasi-linear settings with public budgets," Economics Letters, 115, 100-103.

Ma, H., R. Meir, And D. C. PArkes (2016): "Social choice for agents with general Utilities," in Proceedings of the 25th International Joint Conference on Artificial Intelligence (IJCAI'16), 345-351.

Milgrom, P. And I. Segal (2002): "Envelope theorems for arbitrary choice sets," Econometrica, 70, 583-601.

Mishra, D., A. Pramanik, And S. Roy (2014): "Multidimensional mechanism design in single peaked type spaces," Journal of Economic Theory, 153, 103-116.

Mishra, D. And S. Roy (2013): "Implementation in multidimensional dichotomous domains," Theoretical Economics, 8, 431-466.

Morimoto, S. And S. SERIZAWA (2015): "Strategy-proofness and efficiency with nonquasi-linear preferences: A characterization of minimum price Walrasian rule," Theoretical Economics, 10, 445-487.

Carbajal, J.C. And A. Mualem (2017): "Revenues and budgets: Selling mechanisms for financially constraint buyers," Working Paper.

Muller, R., A. Perea, And S. Wolf (2007): "Weak monotonicity and Bayes-Nash incentive compatibility," Games and Economics Behavior, 61, 344-358.

Myerson, R. B. (1981): "Optimal auction design," Mathematics of Operations Research, 6, 58-73. 
Myerson, R. B. And M. A. Satterthwaite (1983): "Efficient mechanisms for bilateral trading," Journal of Economic Theory, 29, 265-281.

Noldeke, G. And L. Samuelson (2015): "The implementation duality," Cowles Foundation Discussion Paper.

PAI, M. M. AND R. Vohra (2014): "Optimal auctions with financially constrained buyers," Journal of Economic Theory, 150, 383-425.

Rochet, J. C. (1987): "A necessary and sufficient condition for rationalizability in a quasilinear context," Journal of Mathematical Economics, 16, 191-200.

Rockafellar, R. T. (1970): Convex analysis, Princeton University Press, Princeton, New Jersey.

Saitoh, H. And S. Serizawa (2008): "Vickrey allocation rule with income effect," Economic Theory, 35, 391-401.

SAKAI, T. (2008): "Second price auctions on general preference domains: Two characterizations," Economic Theory, 37, 347-356.

- (2013a): "Axiomatizations of second price auctions with a reserve price," International Journal of Economic Theory, 9, 255-265.

_ (2013b): "An equity characterization of second price auctions when preferences may not be quasilinear," Review of Economic Design, 17, 17-26.

SAKS, M. E. AND L. YU (2005): "Weak monotonicity suffices for truthfulness on convex domains," in Proceedings of $7^{\text {th }}$ ACM Conference on Electronic Commerce, ACM Press, $286-293$.

Thomson, W. (2016): "The theory of fair allocation," Forthcoming, Princeton University Press, Princeton, New Jersey.

Vickrey, W. (1961): "Counterspeculation, auctions, and competitive sealed tenders," Journal of Finance, 16, 8-37.

Vohra, R. V. (2011): Mechanism design: A linear programming approach, Cambridge University Press. 
YAMAShita, T. (2015): "Implementation in weakly undominated strategies: Optimality of second-price auction and posted-price mechanism," The Review of Economic Studies, 82, $1223-1246$.

Zhou, Y. And S. Serizawa (2016): "Strategy-proofness and efficiency for non-quasi-linear common-tiered-object preferences: Characterization of minimum price rule," ISER Discussion paper No. 971. 


\section{Appendix B: Optimal mechanism with many agents}

In this appendix, we explore how to extend the one-agent optimal mechanism analysis of Section 6.2 to the multiple agent case. There are two difficulties in extending the analysis of the one agent model.

1. First, the two parameters $\tau_{i}^{0}$ and $\tau_{i}^{1}$ will now depend on the preferences of agents other than $i$ - note that these are infinite dimensional objects. As a result, the onedimensionality nature of the problem in the one-agent case (Section 6.2) is lost. This makes optimization difficult. To partially overcome this intractability issue, we focus attention on a simpler class of mechanisms that we call WP-mechanisms and make some observations about optimality inside this class.

2. The second problem with the multi-agent case is the payment to losing agents. To remind, the analysis in Section 6.2 became tractable because the payment of the losing agent (i.e., if an agent does not get the object) could be fixed to zero by Theorem 5 . The technique of Theorem 5 does not extend to the multiple agents case because when there are more than one agent, the new mechanism constructed in Theorem 5 may not necessarily be feasible. Since the losing agents' payment cannot be fixed at zero, it adds new problems to the optimization.

Below, we focus on a restricted class of mechanisms and show that standard Myersonian techniques can be extended inside this class. This allows us to make some observations about the nature of the expected revenue maximizing mechanism.

We say two type profiles $\mathbf{R}$ and $\mathbf{R}^{\prime}$ are $\mathbf{W P}$-equivalent at $t$ if $W P\left(R_{i}, t\right)=W P\left(R_{i}^{\prime}, t\right)$ for all $i \in N$. The following class of mechanisms fixes the payment of losing agents at some value and then elicits only the WP values of agents at that payment.

Definition 21 A mechanism $(f, \mathbf{p})$ is a WP-mechanism if there exists a real number $\tau^{0}$ such that

1. $f_{i}(\mathbf{R})=0$ implies $p_{i}(\mathbf{R})=\tau^{0}$ for all $\mathbf{R}$ and for all $i \in N$,

2. $f_{i}(\mathbf{R})=f_{i}\left(\mathbf{R}^{\prime}\right)$ and $p_{i}(\mathbf{R})=p_{i}\left(\mathbf{R}^{\prime}\right)$ for all $i \in N$ if $\mathbf{R}$ and $\mathbf{R}^{\prime}$ are WP-equivalent at $\tau^{0}$.

By Theorem 7, every WP-mechanism must announce a $\tau^{0}$ (the amount a losing agent pays), and determines the allocation and transfer amounts based on the WP values of agents at $\tau^{0}$. Given a value of $\tau^{0}$ and a type profile $\mathbf{R}$, we denote the profile of $W P\left(R_{1}, \tau^{0}\right), \ldots, W P\left(R_{n}, \tau^{0}\right)$ 
as $\mathbf{w}\left(\tau^{0}\right)$. For simplicity, we denote by $f_{i}\left(\mathbf{w}\left(\tau^{0}\right)\right)$ the allocation $f_{i}(\mathbf{R})$ and by $p_{i}\left(\mathbf{w}\left(\tau^{0}\right)\right)$ the transfer $p_{i}(\mathbf{R})$.

By Theorem 7, the payment of agent $i$ at this type profile is

$$
p_{i}\left(\mathbf{w}\left(\tau^{0}\right)\right)=\tau^{0}+\tau_{i}^{1}\left(w_{-i}\left(\tau^{0}\right)\right) f_{i}\left(\mathbf{w}\left(\tau^{0}\right)\right)
$$

We need to compute the expected payment of each agent from a mechanism. For this, we associate priors on the WP values at $\tau^{0}$. We assume that for every $t \in \mathbb{R}$, the WP values at $t$ are drawn from some absolutely continuous distribution $G(\cdot ; t)$ with density $g(\cdot ; t)$ from a support $(0, \beta)$, where $\beta \in \mathbb{R}_{++} \cup\{\infty\}$ - note that the support of WP values is independent of $t .{ }^{13}$ We also assume that $G(\cdot ; t)$ is regular in the sense that for all $w>w^{\prime}$ and for all $t \leq 0$, we have

$$
\frac{g(w ; t)}{1-G(w ; t)} \geq \frac{g\left(w^{\prime} ; t\right)}{1-G(w ; t)} .
$$

Using such a distribution, we compute the expected payment of agent from a WPmechanism $(f, \mathbf{p})$ and denote it as $\rho_{i}(f, \mathbf{p})$.

A mechanism $(f, \mathbf{p})$ is individually rational if for every $i \in N$ and every $\mathbf{R}$, we have $\left(f_{i}(\mathbf{R}), p_{i}(\mathbf{R})\right) \geq 0$. The proof of Lemma 1 can be replicated to show that a DSIC mechanism $(f, \mathbf{p})$ is individually rational if and only if the payment of losing agents is non-positive, i.e., for a WP-mechanism at $\tau^{0}$, we must have $\tau^{0} \leq 0$.

A WP-mechanism $(f, \mathbf{p})$ is an optimal WP-mechanism if it is DSIC and individually rational, and for every DSIC and individually rational WP-mechanism $\left(f^{\prime}, \mathbf{p}^{\prime}\right)$ we have

$$
\sum_{i \in N} \rho_{i}(f, \mathbf{p}) \geq \sum_{i \in N} \rho_{i}\left(f^{\prime}, \mathbf{p}^{\prime}\right) .
$$

We provide a partial description of the optimal WP-mechanism below.

THEOREM 8 An optimal WP-mechanism satisfies the following. There exists a non-positive number $\tau^{0} \leq 0$ such that

- each agent is paid a subsidy $-\tau^{0} \geq 0$ before he announces his type,

- a reserve price $r=\phi^{-1}(0)$ is announced, where $\phi$ is the map $\phi(w)=w-\frac{1-G\left(w ; \tau^{0}\right)}{g\left(w ; \tau^{0}\right)}$ for all $w$,

- for all $\mathbf{R}$,

\footnotetext{
13 This assumption and the symmetry assumption can be changed without changing the qualitative nature of the results below.
} 
- the object is not allocated and no transfers are made if $W P\left(R_{i}, \tau^{0}\right)<r \forall i \in N$;

- else it is allocated to an agent with highest $W P\left(\cdot ; \tau^{0}\right)$ value.

In the latter case, the winning agent $i$ pays an amount equal to $\max \left(r, \max _{j \neq i} W P\left(R_{j}, \tau^{0}\right)\right)$.

Essentially, Theorem 8 says that the optimal WP-mechanism is the standard Vickrey auction with the appropriate reserve price, but with two significant differences: (1) some subsidy $-\tau^{0}$ may have to be given to all the agents before the start of the auction; and (2) the Vickrey auction with reserve price is conducted with respect to the willingness to pay at $W P\left(\cdot, \tau^{0}\right)$.

Below, we provide a proof of Theorem 8 - once we use Theorem 7 , it follows standard Myersonian techniques. The computation of optimal value of $\tau^{0}$ will depend on the particulars of the model. It seems unlikely that a particular recommendation of $\tau^{0}$ can be made for a general enough class of models.

\section{Proof of Theorem 8}

Proof: A consequence of our result in Theorem 7 is that a DSIC and IR WP-mechanism is completely described by a real number $\tau^{0} \leq 0$ and the allocation rule $x$ (which only depends on the WP values at $\tau^{0}$ ).

Fix any such WP-mechanism that satisfies $\tau^{0} \leq 0$. For simplicity, we will denote the WP of an agent $i$ at the specified $\tau^{0}$ as $w_{i}$. At a profile of $\mathrm{WP} \mathbf{w}$, the payment of agent $i$ is given by

$$
p_{i}(\mathbf{w})=\tau^{0}+\tau_{i}^{1}\left(w_{-i}\right) f_{i}(\mathbf{w})=\tau^{0}+w_{i} f_{i}(\mathbf{w})-\int_{0}^{w_{i}} f_{i}\left(y_{i}, w_{-i}\right) d y_{i} .
$$

Hence, expected payment of agent $i$ with WP $w_{i}$ is given by

$$
\begin{aligned}
E P\left(w_{i} ; \tau^{0}\right) & =\tau^{0}+w_{i} \int f_{i}\left(w_{i}, w_{-i}\right) g_{-i}\left(w_{-i} ; \tau^{0}\right) d w_{-i}-\int_{0}^{w_{i}} \int f_{i}\left(y_{i}, w_{-i}\right) g_{-i}\left(w_{-i} ; \tau^{0}\right) d w_{-i} d y_{i} . \\
& =\tau^{0}+w_{i} E F\left(w_{i} ; \tau^{0}\right)-\int_{0}^{w_{i}} E F\left(y_{i} ; \tau^{0}\right) d y_{i},
\end{aligned}
$$

where $E F\left(y_{i} ; \tau^{0}\right):=\int f_{i}\left(y_{i}, w_{-i}\right) g_{-i}\left(w_{-i} ; \tau^{0}\right) d w_{-i}$ for all $y_{i}$. Hence, expected revenue from 
agent $i$ is given by

$$
\begin{aligned}
& \tau^{0}+\int_{0}^{\beta} w_{i} E F_{i}\left(w_{i} ; \tau^{0}\right) g_{i}\left(w_{i} ; \tau^{0}\right) d w_{i}-\int_{0}^{\beta} \int_{0}^{w_{i}} E F_{i}\left(y_{i} ; \tau^{0}\right) d y_{i} g_{i}\left(w_{i} ; \tau^{0}\right) d w_{i} . \\
& =\tau^{0}+\int_{0}^{\beta} w_{i} E F_{i}\left(w_{i} ; \tau^{0}\right) g_{i}\left(w_{i} ; \tau^{0}\right) d w_{i}-\int_{0}^{\beta} \int_{w_{i}}^{\beta} g_{i}\left(y_{i} ; \tau^{0}\right) d y_{i} E F_{i}\left(w_{i} ; \tau^{0}\right) d w_{i} . \\
& =\tau^{0}+\int_{0}^{\beta} w_{i} E F_{i}\left(w_{i} ; \tau^{0}\right) g_{i}\left(w_{i} ; \tau^{0}\right) d w_{i}-\int_{0}^{\beta}\left(1-G_{i}\left(w_{i} ; \tau^{0}\right)\right) E F_{i}\left(w_{i} ; \tau^{0}\right) d w_{i} \\
& =\tau^{0}+\int_{0}^{\beta}\left(w_{i}-\frac{1-G_{i}\left(w_{i} ; \tau^{0}\right)}{g_{i}\left(w_{i} ; \tau^{0}\right)}\right) E F_{i}\left(w_{i} ; \tau^{0}\right) g_{i}\left(w_{i} ; \tau^{0}\right) d w_{i} \\
& =\tau^{0}+\int_{0}^{\beta} \phi\left(w_{i} ; \tau^{0}\right) E F_{i}\left(w_{i} ; \tau^{0}\right) g_{i}\left(w_{i} ; \tau^{0}\right) d w_{i},
\end{aligned}
$$

where $\phi\left(w_{i} ; \tau^{0}\right):=w_{i}-\frac{1-G_{i}\left(w_{i} ; \tau^{0}\right)}{g_{i}\left(w_{i} ; \tau^{0}\right)}$ for all $w_{i}$. Hence, expected revenue from the mechanism is given by

$$
\begin{aligned}
& \sum_{i \in N} \tau^{0}+\sum_{i \in N} \int_{0}^{\beta} \phi\left(w_{i} ; \tau^{0}\right) E F_{i}\left(w_{i} ; \tau^{0}\right) g_{i}\left(w_{i} ; \tau^{0}\right) d w_{i} \\
& =n \tau^{0}+\sum_{i \in N} \int_{0}^{\beta} \phi\left(w_{i} ; \tau^{0}\right) \int f_{i}\left(w_{i}, w_{-i}\right) g_{-i}\left(w_{-i} ; \tau^{0}\right) d w_{-i} g_{i}\left(w_{i} ; \tau^{0}\right) d w_{i} \\
& =n \tau^{0}+\int\left(\sum_{i \in N} \phi\left(w_{i} ; \tau^{0}\right) f_{i}\left(w_{i}, w_{-i}\right)\right) g\left(\mathbf{w} ; \tau^{0}\right) d \mathbf{w} .
\end{aligned}
$$

For a fixed $\tau^{0} \leq 0$, the second term in the above expression is maximized by the standard Myersonian argument (under regularity, i.e., ) by maximizing virtual values - this leads us the standard optimal mechanism in the symmetric case, which is a second price Vickrey auction with a reserve price equal to $\phi^{-1}(0)$. This establishes the result. 